\title{
The FAMU experiment at RAL to study the muon transfer rate from hydrogen to other gases
}

\section{FAMU collaboration}

amczak, ${ }^{a}$ G. Baccolo ${ }^{b, r}$ S. Banfi, ${ }^{b, q}$ D. Bakalov, ${ }^{c}$ G. Baldazzi, ${ }^{d}$ R. Benocci, ${ }^{b, r}$

R. Bertoni, ${ }^{b}$ M. Bonesini, ${ }^{b, q, 1}$ V. Bonvicini, ${ }^{e}$ F. Chignoli, ${ }^{b}$ M. Clemenza, ${ }^{b, q}$ L. Colace, ${ }^{h, i}$

M. Danailov, ${ }^{e, u}$ P. Danev, ${ }^{c}$ A. de Bari, ${ }^{f, g}$ C. De Vecchi, ${ }^{g}$ M. De Vincenzi, ${ }^{h, j}$ E. Furlanetto, $, e, p$

F. Fuschino, ${ }^{d, x}$ K. Gadejisso-Tossou, ${ }^{e, k, t}$ D. Guffanti, ${ }^{e, w}$ A. laciofano, ${ }^{h}$ K. Ishida, ${ }^{l}$

C. Labanti, ${ }^{d, x}$ V. Maggi, ${ }^{b, r}$ A. Margotti, ${ }^{d}$ R. Mazza, ${ }^{b}$ A. Menegolli, ${ }^{f, g}$ E. Mocchiutti, ${ }^{e}$

M. Moretti, ${ }^{b, r}$ G. Morgante, ${ }^{d, x}$ M. Nastasi, ${ }^{b, q}$ J. Niemela, ${ }^{k}$ C. Pizzolotto, ${ }^{e}$ E. Previtali, ${ }^{b,} q$

A. Pullia, ${ }^{m, n}$ R. Ramponi, ${ }^{m, s}$ A. Rachevski, ${ }^{e}$ L. P. Rignanese, ${ }^{d}$ M. Rossella, ${ }^{g}$ N. Rossi, ${ }^{p}$

R. Sarkar, ${ }^{e, y}$ M. Stoilov, ${ }^{c}$ L. Stoychev, ${ }^{e, k}$ A. Tomaselli, ${ }^{g, o}$ L. Tortora, ${ }^{h}$ E. Vallazza, ${ }^{e}$ G. Zampa ${ }^{e}$ and A. Vacchi, ${ }^{e, l, p}$

${ }^{a}$ Institute of Nuclear Physics, Polish Academy of Sciences, Radzikowskiego 152, PL31342 Kraków, Poland

${ }^{b}$ Sezione INFN di Milano Bicocca, Piazza della Scienza 3, Milano, Italy

${ }^{c}$ Institute for Nuclear Research and Nuclear Energy, Bulgarian Academy of Sciences, blvd. Tsarigradsko ch. 72, Sofia 1142, Bulgaria

${ }^{d}$ Sezione INFN di Bologna, viale Berti Pichat 6/2, Bologna, Italy

e Sezione INFN di Trieste, via A. Valerio 2, Trieste, Italy

${ }^{f}$ Dipartimento di Fisica, Università di Pavia, via Bassi 6, Pavia, Italy

${ }^{3}$ Sezione INFN di Pavia, Via A. Bassi 6, Pavia, Italy

${ }^{h}$ Sezione INFN di Roma Tre, Via della Vasca Navale 84, Roma, Italy

${ }^{i}$ Dipartimento di Ingegneria, Università degli Studi Roma Tre, Via V. Volterra 62, Roma, Italy

${ }^{j}$ Dipartimento di Matematica e Fisica, Università di Roma Tre, Via della Vasca Navale 84, Roma, Italy

${ }^{k}$ The Abdus Salam International Centre for Theoretical Physics, Strada Costiera 11, Trieste, Italy

${ }^{l}$ Riken Nishina Center, RIKEN, 2-1 Hirosawa, Wako, Saitama 351-0198, Japan

${ }^{m}$ Sezione INFN di Milano, via Celoria 16, Milano, Italy

${ }^{n}$ Dipartimento di Fisica, Università degli Studi di Milano, via Celoria 16, Milano, Italy

o Dipartimento di Ingegneria, Università di Pavia, Via Ferrata 5, Pavia, Italy

${ }^{p}$ Dipartimento di Scienze Matematiche, Informatiche e Fisiche, Università di Udine, via delle Scienze 206, Udine, Italy

${ }^{q}$ Dipartimento di Fisica G. Occhialini, Università di Milano Bicocca, Piazza Scienza 3, Milano, Italy

${ }^{r}$ Dipartimento di Scienze dell’Ambiente e della Terra, Università di Milano Bicocca, Piazza Scienza 1, 20126 Milano, Italy

${ }^{s}$ IFN-CNR, Dipartimento di Fisica, Politecnico di Milano, piazza Leonardo da Vinci 32, Milano, Italy

${ }^{1}$ corresponding author 
${ }^{t}$ Laboratoire de Physique des Composants à Semi-conducteurs (LPCS), Department de physique, Université de Lomé, Lomé, Togo

"Sincrotrone Elettra Trieste, SS14, km 163.5, Basovizza, Italy

${ }^{w}$ Gran Sasso Science Institute, via F. Crispi 7, L' Aquila, Italy

${ }^{x}$ INAF-IASF Bologna, Area della Ricerca, via P. Gobetti 101, Bologna, Italy.

${ }^{y}$ Indian Centre for Space Physics, 43 Chalantika, Garia Station Road, Garia, Kolkata, 700084 West Bengal, India

E-mail: maurizio.bonesini@mib.infn.it

Abstract: The aim of the FAMU (Fisica degli Atomi Muonici) experiment is to realize the first measurement of the hyperfine splitting (hfs) in the $1 \mathrm{~S}$ state of muonic hydrogen $\Delta E_{1 S}^{h f s}$, by using the RIKEN-RAL intense pulsed muon beam and a high-energy mid-infrared tunable laser. This requires a detailed study of the muon transfer mechanism at different temperatures and hence at different epithermal states of the muonic system. The experimental setup involves a cryogenic pressurized gas target and a detection system based on silicon photomultipliers-fiber beam hodoscopes, high purity Germanium detectors and Cerium doped Lanthanium Bromide crystals for X-rays detection at energies around $100 \mathrm{keV}$.

Simulation, construction and detector performances of the FAMU apparatus at RAL are reported in this paper.

This is version v13 - to be submitted to JINST.

KeYwords: Muonic atoms; Detection systems; Precision spectroscopy 


\section{Contents}

1 Introduction 1

2 The FAMU experimental setup $\quad 2$

2.1 The $1 \mathrm{~mm}$ pitch beam hodoscope 4

2.2 The cryogenic target system 4

$\begin{array}{lll}\text { 2.2.1 Cooling system } & 7\end{array}$

2.3 The $\mathrm{Ce}: \mathrm{LaBr}_{3} \mathrm{X}$-ray detectors with PMT readout 8

2.4 Compact X-rays detectors with SiPM array readout 9

2.5 The HPGe X-ray detectors 9

2.6 Beam momentum tuning and detectors positioning 10

3 Raw data handling $\quad 11$

$\begin{array}{lll}3.1 & \text { Structure of offline data processing } & 14\end{array}$

4 Experimental operations and performances $\quad 16$

$\begin{array}{lll}4.1 & \text { Target operations } & 17\end{array}$

4.2 Beam characterisation with the $1 \mathrm{~mm}$ pitch hodoscope 18

4.3 Detection of characteristic X-rays with Ce:LaBr 3 with PMT readout 19

4.4 Detection of characteristic X-rays with crystals with SiPM array readout 21

4.5 Detection of characteristic X-rays with HPGe detectors 23

5 Conclusions 25

\section{Introduction}

The FAMU proposal $[1,2]$ aims to a new high precision measurement of muonic Hydrogen spectroscopy. In this experiment, incident low momentum muons from pulsed muon source are stopped in a Hydrogen gas mixture at appropriate temperature and pressure to form muonic Hydrogen atoms $\mu p$. Once the thermal $\mu p$ have reached the ground para $(\mathrm{F}=0)$ state, they are exposed to photons of energy equal to the hyperfine-splitting resonance-energy $\Delta E^{h f s} \simeq 0.182 \mathrm{eV}$, and excited to the ortho $(\mathrm{F}=1)$ spin state. They then very quickly de-excite to the $(\mathrm{F}=0)$ state in subsequent collisions with the surrounding $\mathrm{H}_{2}$ molecules [3]. At the exit of the collision the muonic atom is accelerated, because of energy and momentum conservation, by $2 / 3$ of the excitation energy $\Delta E^{h f s}$.

In the following thermal collisions of $\mu p$, part of the muons are transferred to a low concentration admixed gas $Z$, forming excited states of $\mu Z$. The rate of this reaction depends on the kinetic energy of the muonic atoms.

By tuning the emission wavelength of the laser around $\Delta E^{h f s} \simeq 0.182 \mathrm{eV}$, corresponding to $6.78 \mu \mathrm{m}$, it is possible to experimentally determine the number of muonic atoms that have 


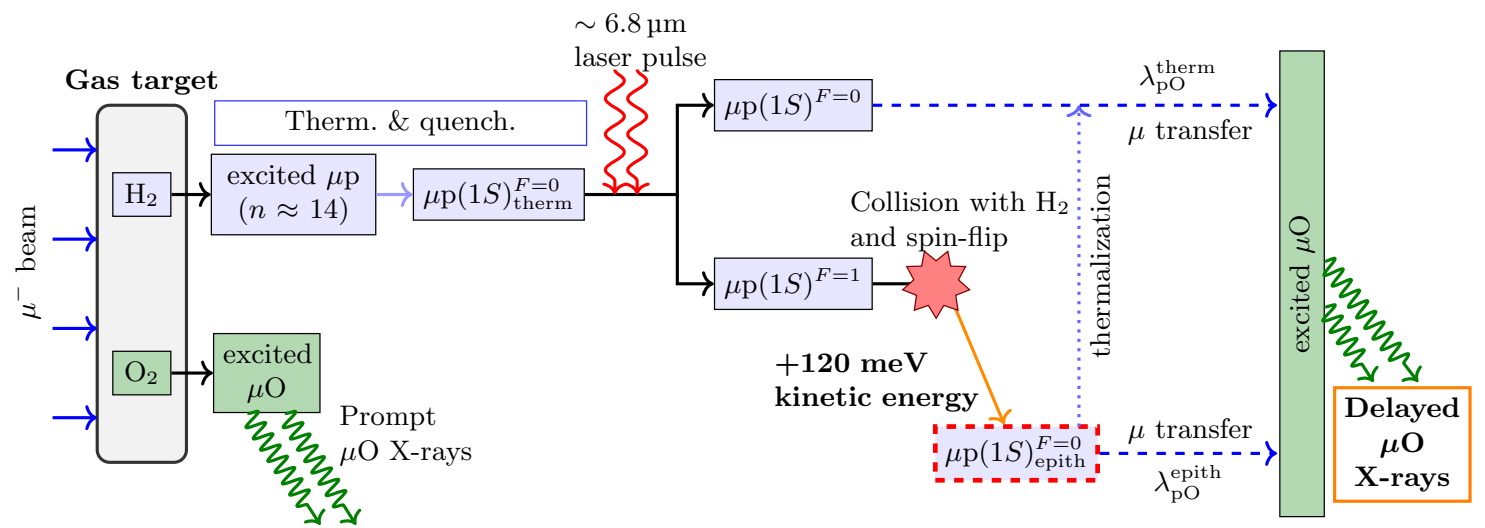

Figure 1. Schematic representation of the employed experimental method. Here Oxygen is the admixed gas Z.

undergone the above sequence of processes, and identify the resonance wavelength as the value for which the number of spin-excited atoms is maximal. As outlined in figure 1, the muonic Hydrogen atoms are created and then propagate in a gas target with an appropriate mixture of Hydrogen and a higher-Z contaminant. The observable is the time distribution of the characteristic X-ray emitted from the muonic atoms produced by muon transfer from Hydrogen to the atom of the admixture gas $(\mu p)+Z \rightarrow \mu Z^{*}+p$ and its response to variations of the laser excitation wavelength $[4,5]$. These X-rays are distinguished by the prompt X-ray from muonic atoms, formed in direct prompt capture, by the time delay with respect to the beam arrival. The $\left(\mu^{-} p\right)_{1 S}$ hfs resonance is recognized by the maximal response to the tuned laser wavelength of the time distribution of X-ray K-lines from $(\mu Z)^{*}$ cascade, i.e. by the maximal difference between the time distributions in presence and without laser radiation.

\section{The FAMU experimental setup}

The pulsed muon source of the RIKEN muon facility at the Rutherford Appleton Laboratory (RAL) [6], is well suited to the purpose of the project. It can deliver about 3 to $8 \times 10^{4}$ negative muons per second with a pulse repetition rate of $50 \mathrm{~Hz}$ and momentum in the range $30-80 \mathrm{MeV} / \mathrm{c}$, with $\sigma_{p} / p=4 \%$ and a beam transverse section of $\sigma_{x}, \sigma_{y}=1.5 \mathrm{~cm}$. The beam has a double peak structure with $70 \mathrm{~ns}$ pulse width (FWHM) and peak to peak distance of $320 \mathrm{~ns}$. The beam can be delivered alternatively to four experimental ports.

The progression of the FAMU project has been planned into several steps. The first step took data in June 2014 [7], with a preliminary setup and allowed to publish FAMU first results on muon transfer rate to the heavier admitted gas $[8,9]$. It has been followed by the experiment described in the detail in this paper, that took data from 2016 on. The setup with the laser will be implemented in a future phase. The experimental apparatus of the FAMU experiment is based on:

- a $1 \mathrm{~mm}$ pitch X/Y beam hodoscope to tune the beam steering onto the target and evaluate the muon incoming rate, thus optimizing the efficiency of the data taking;

- a cryogenic target, where Hydrogen gas admixed with an heavier contaminant is contained; 


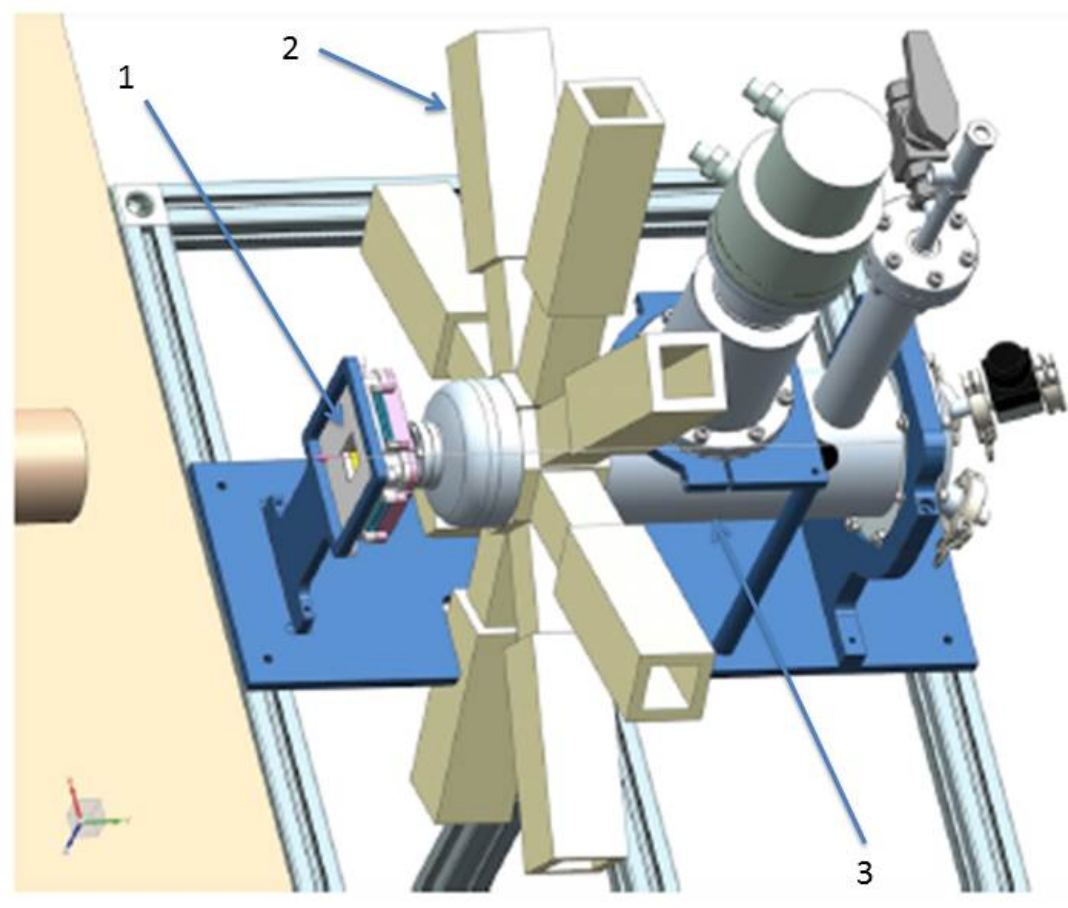

Figure 2. Lateral artist view of the FAMU experiment at RIKEN-RAL. The beam comes from the left. Visible are the $1 \mathrm{~mm}$ pitch beam hodoscope (1), the Ce: $\mathrm{LaBr}_{3}$ crystals with photomultiplier (PMT) readout (2), arranged in a crown of 8 elements and the cryogenic target (3). The HPGe detectors are not drawn in this view.

- a system for characteristic X-rays detection, based mainly on 1" thick cylindrical Cerium doped Lanthanium Bromide(Ce: $\left.\mathrm{LaBr}_{3}\right)$ crystals and High Purity Germanium (HPGe) detectors.

A schematic layout is shown in figure 2. The apparatus described here was used for four runs, of which the February 2016 one was the main data taking period.

In detail, the main goals of the 2016 data taking were:

- measure the muon transfer rate from muonic Hydrogen to Oxygen and other gases at different temperatures (between 300 and $100 \mathrm{~K}$ ), pressures and concentration;

- validate the theoretical calculation about the best Oxygen concentration;

- study different contaminants which could give a temperature (energy) muon transfer rate dependence as Oxygen;

- perform background tests with empty target or filled with pure Hydrogen or pure Nitrogen.

The collected runs, taken in February 2016, are resumed in table 1. 
Table 1. Data sets taken in the 2016 FAMU experiment at RIKEN-RAL.

\begin{tabular}{|l|c|c|c|c|c|}
\hline gas mixture & $\begin{array}{c}p_{\text {beam }} \\
(\mathrm{MeV} / \mathrm{c})\end{array}$ & temp $\left({ }^{0} \mathrm{~K}\right)$ & data taking & goals & filling at \\
\hline \hline $\mathrm{N}_{2}(100 \%)$ & 57 & 298 & $6 \mathrm{~h}$ & det. calib/test & 2 bar $300 \mathrm{~K}$ \\
\hline $\mathrm{H}_{2}+\mathrm{O}_{2}(0.3 \%)$ & 57 & $\begin{array}{l}300 / 273 / 240 / \\
200 / 150 / 100\end{array}$ & $3 \mathrm{~h} / \mathrm{step}$ & thermal cycle & 41 bar $300 \mathrm{~K}$ \\
\hline$H_{2}+\mathrm{O}_{2}(0.3 \%)$ & 57 & 100 & $20 \mathrm{~m}$ & high p/low T test & 30 bar $100 \mathrm{~K}$ \\
\hline$H_{2}+\mathrm{O}_{2}(0.05 \%)$ & 57 & $300 / 273 / 240$ & $3 \mathrm{~h} / \mathrm{step}$ & thermal cycle & 41 bar $300 \mathrm{~K}$ \\
\hline$H_{2}+\mathrm{Ar}(0.3 \%)$ & 57 & $300 / 273 / 240 /$ & $3 \mathrm{~h} / \mathrm{step}$ & thermal cycle & 41 bar $300 \mathrm{~K}$ \\
& & $200 / 150 / 100$ & & & \\
\hline Vacuum & 57 & - & $3 \mathrm{~h}$ & backg studies & $\approx 10^{-6} \mathrm{mbar}$ \\
\hline $\mathrm{H}_{2}+\mathrm{Ar}(1 \%)$ & 57 & $300 / 273 / 240$ & $3 \mathrm{~h} / \mathrm{step}$ & thermal cycle & 41 bar $300 \mathrm{~K}$ \\
\hline $\mathrm{H}_{2}+\mathrm{O}_{2}(1 \%)$ & 57 & $300 / 273 / 240$ & $3 \mathrm{~h} / \mathrm{step}$ & thermal cycle & 41 bar $300 \mathrm{~K}$ \\
\hline $\mathrm{H}_{2}(100 \%)$ & 57 & 300 & $30 \mathrm{~m}$ & backg studies & 41 bar $300 \mathrm{~K}$ \\
\hline$H_{2}+\mathrm{O}_{2}(0.3 \%)$ & 57 & 300 & $30 \mathrm{~m}$ & check 2014 results & 41 bar $300 \mathrm{~K}$ \\
\hline $\mathrm{H}_{2}+\mathrm{CH}_{4}(0.3 \%)$ & 57 & $300 / 273 / 240 /$ & $3 \mathrm{~h} / \mathrm{step}$ & thermal cycle & 41 bar $300 \mathrm{~K}$ \\
& & 200 & & & \\
\hline
\end{tabular}

\subsection{The $1 \mathrm{~mm}$ pitch beam hodoscope}

A relevant issue for the FAMU experiment is the optimal steering of the high intensity pulsed muon beam impinging onto the Hydrogen target, to maximize the muonic Hydrogen production rate. A system of three beam hodoscopes based on square scintillating fibers read by silicon photomultipliers (SiPM) has been developed for this scope: two have a $3 \mathrm{~mm}$ pitch and are removable and are used only for special runs [10], while the last has a $1 \mathrm{~mm}$ pitch and is permanently installed in front of the target entrance [11].

To reduce the amount of material in front of the target entrance window, $1 \mathrm{~mm}^{2}$ square scintillating Bicron BCF12 fibers coated with white EMA, to avoid light cross-talk, were used. The fibers $(32+32)$ were arranged in parallel on two orthogonal planes along $\mathrm{X} / \mathrm{Y}$ coordinates, giving a detector active area of $32 \times 32 \mathrm{~mm}^{2}$. RGB SiPMs from Advansid, with a total $1 \times 1 \mathrm{~mm}^{2}$ active area square with $40 \mu \mathrm{m}$ cells, were used to detect scintillation light. Because the SiPM's footprint is slightly bigger than the fiber cross-section, fibers had to be read alternating left/right and up/down sides. All available SiPMs were tested individually to determine their breakdown voltages by measuring their current-voltage characteristic curve. It was possible, with a suitable selection of the SiPMs to be used, to employ a common voltage for the biasing of the SiPMs of each detector side. The SiPM's signals are then fed into a $5 \mathrm{~Gb} / \mathrm{s}$ CAEN V1742 FADC and processed via the standard FAMU DAQ system based on a CAEN V2718 VME-PCI interface. Onboard 100 $\mathrm{k} \Omega$ SMD thermistors have been mounted to ensure long-term stability via offline correction of the thermal drift of SiPMs' gain. Some details of the detector mounting are shown in figure 3.

\subsection{The cryogenic target system}

The efficiency of the method proposed by the FAMU collaboration depends on the collisional energy dependence of the muon transfer rate. While for many gases the muon transfer rate from the $\mu p$ system at low energies is nearly constant, there is experimental evidence that for Oxygen there is instead a sharp energy dependence [12]. Monte Carlo simulations, based on these data, have 

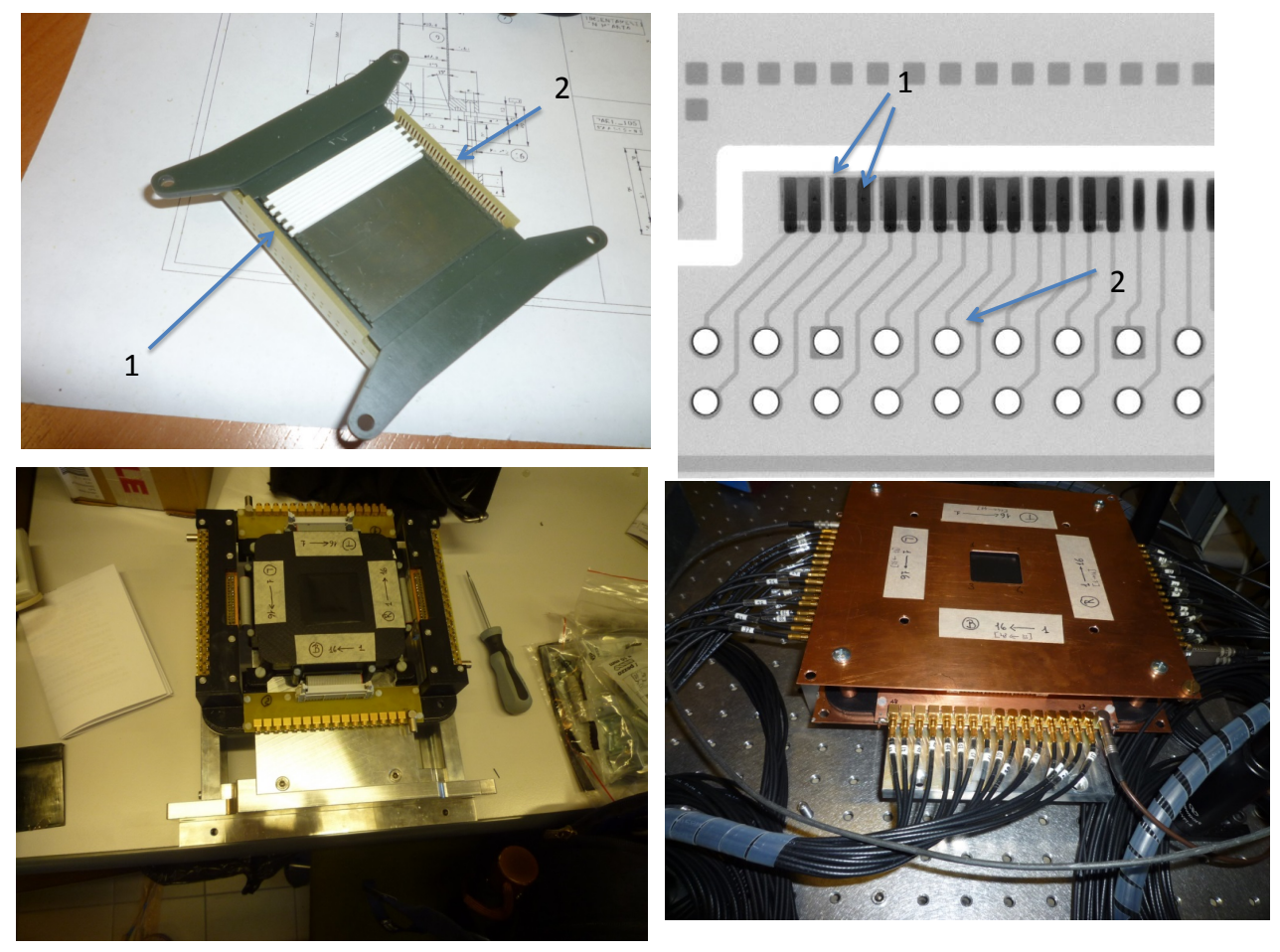

Figure 3. Left-top panel: holder of the $1 \mathrm{~mm}$ square fibers. Visible are some white coated scintillating fibers (1) and the printed circuit board(PCB) (2), where SiPM are soldered. Right top panel: X-rays image to cross-check the SiPMs mounting on the PCB. (1) are the conductive tracks, where cathode and anode of a SiPM are soldered; (2) is the footprint where the 40-way connector for flat cable is mounted. Left-bottom panel: mounted detector, with an interface board visible. Signals cables are to be attached to the row of MCX connectors. Right-bottom panel: complete detector, with two $1.5 \mathrm{~mm}$ copper plates installed for electrical shielding.

shown that the method proposed by FAMU may provide the expected results [5]. Its experimental verification requires a detailed study of the muon transfer mechanism at different temperatures and hence at different epithermal state of the muonic system.

The cryogenic gas target system was developed for this task with the following characteristics:

- low-mass beam entrance window for minimal losses and lateral spread of the impinging muon beam;

- high transparency of the target lateral walls to the X-rays of the muonic lines of interest;

- capability to held pressures up to 40 atm of ultrapure Hydrogen gas;

- ability to work at stable temperatures from 50 to $300 \mathrm{~K}$.

The resulting target design ${ }^{1}$ is shown in the left panel of figure 4. An Aluminium alloy cryostat insulates the internal target, containing the gas, from the environment by means of a vacuum jacket,

${ }^{1}$ Realized by Criotec Impianti srl [13]. 

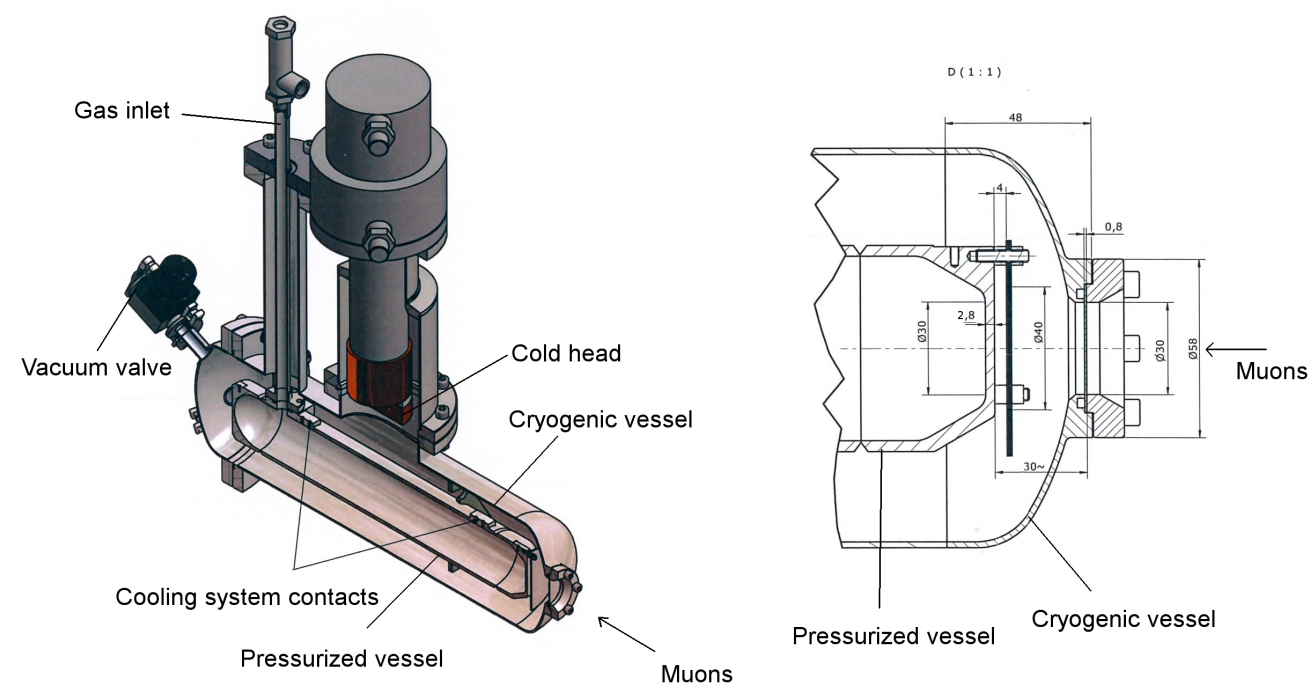

Figure 4. The target design. Left panel: the double volume shell needed for thermal insolation is clearly visible: the whole is realized in Aluminium alloy. Right panel: the details of the muon beam entrance window. Beam goes from right to left.

MLI superinsulation shrouds and fiberglass stunts. In the left panel of figure 4, from right to left muons will cross the first thin $0.8 \mathrm{~mm}$ Aluminium entrance window entering the evacuated volume, where a stack of three Aluminium disks $(0.1 \mathrm{~mm}$ each) with fiberglass ring spacers, are used as radiative shields, before entering the pressurized volume through a second $2.8 \mathrm{~mm}$ Aluminium entrance window. The details of the muon beam entrance window are visible in the right panel of figure 4. A careful evaluation of the X-rays background contribution coming from the target has shown the importance of having a thin coating of the internal shell of the target made with high $\mathrm{Z}$ material $(100 \mu \mathrm{m}$ of $\mathrm{Ni}$ and $20 \mu \mathrm{m}$ of $\mathrm{Au})$. This allows fast nuclear capture of the muons reaching the walls, thus reducing the background coming from electrons from muon decay. The cryogenic system has been built and certified to comply with the standard EU safety rules.

The cooling system is built around a Sumitomo CH-104 cold head, coupled to a HC-4E1 helium compressor. It is a single-stage cryogenic refrigerator that operates on the Gifford-McMahon refrigeration cycle, able to provide more than $20 \mathrm{~W}$ at a temperature of $40 \mathrm{~K}$. Water needed for heat extraction at the compressor is supplied by a closed system water chiller with monitored temperature. During the whole data taking the water temperature remained in the range from 8 to 20 degrees Celsius, well within helium compressor specifications.

Four DT-670 Silicon diodes thermometers are integrated in the system: two on the cold head and two at the ends of the inner cylinder. A Lakeshore 336 temperature controller is used to control the target temperature.

Vacuum performances are very good: the system reaches a vacuum level in the $10^{-5}$ 
$-10^{-6}$ mbar range. Even once the thermo-vacuum pump is disconnected (at low temperature) the vacuum level is maintained for several days.

\subsubsection{Cooling system}

The proposed measure of the muon transfer rate requires an efficient operation of the target at various temperatures, with a stability better than $\approx 0.5^{\circ} \mathrm{C}$ and with different gas fillings. The characteristics of the cooling system are described in the following.

The cold head reaches $28 \mathrm{~K}$ in a total of 3.1 hours and stabilizes to its steady-state equilibrium of $27.7 \mathrm{~K}$ in $4-4.5$ hours, showing for the next hours a stability of about $0.06 \mathrm{~K} / \mathrm{h}$. The target follows this trend with a time delay due to its thermal capacitance: it goes below $40 \mathrm{~K}$ in 2.7 hours, reaching $31 \mathrm{~K}$ in $3 \mathrm{~h}$. Two thermometers, on the two ends of the inner cylinder (figure 4), stabilize around $30.5 \mathrm{~K}$ and $31.0 \mathrm{~K}$ respectively for a final average equilibrium temperature of $30.75 \mathrm{~K}$ with nearly 40 minutes delay with respect to the cold head. In this "steady-state" the stability is very good, on the order of $0.01 \mathrm{~K} / \mathrm{h}$. At the minimum equilibrium temperature of the system, with no control power, the temperature difference between the cold head and the target is $3.1 \mathrm{~K}$.

The best approach to save time and have a good temperature stability during system cooling is to perform temperature steps not exceeding $\approx 50 \mathrm{~K}$. With this procedure, a conservative $1 \%$ temperature variation at the required set-point can be used as the threshold for starting the measurement. At the $1 \%$ level we can assume that the transfer rate from muonic Hydrogen to other gases is almost constant. However, being the temperature recorded in the raw data structure, any anomalous effect can be tracked down during the following data analysis. Operatively a set-point is reached when the average temperature of the target is within $1 \%$ of its value and the stability is better than $1 \%$ over $1000 \mathrm{~s}$. The study of transfer rate temperature dependence requires to fix several target temperatures. However it is not needed to set very precisely a given temperature. Thus thermal control can be achieved by picking a set-point value and letting the target stabilize at its natural equilibrium temperature.

Lowering the target temperature relies on refrigerator cooldown capability while heating is based on the power supplied by the Lakeshore controller $(0-100 \mathrm{~W}$ with the presently installed resistor of $25 \Omega$ ). The cooldown rate, as expected, is not constant and it is inversely proportional to the temperature.

Figure 5 shows the target and cold head temperature as function of time during a laboratory test of the cryogenic system. The target is cooled from $250 \mathrm{~K}$ to $205 \mathrm{~K}$. Even if, in this case, the control operations have not been optimized the time needed to cool down the target by $\approx 50 \mathrm{~K}$ is of about one hour.

Thermal operations on the target must also take into account that different gas mixtures and pressure are going to be used. After each cycle the target needs to be correctly evacuated and cleaned. The gas mixture re-loading process has to be done at room temperature, as low temperature condensation and cryo-sorption issues may prevent to reach the required level of cleanliness. Thus, each run with a new gas mixture follows a cooldown profile in steps of $50 \mathrm{~K}$ starting from room temperature (from $300 \mathrm{~K}$ to $100 \mathrm{~K}$ ), as described later in Section 4.1. 


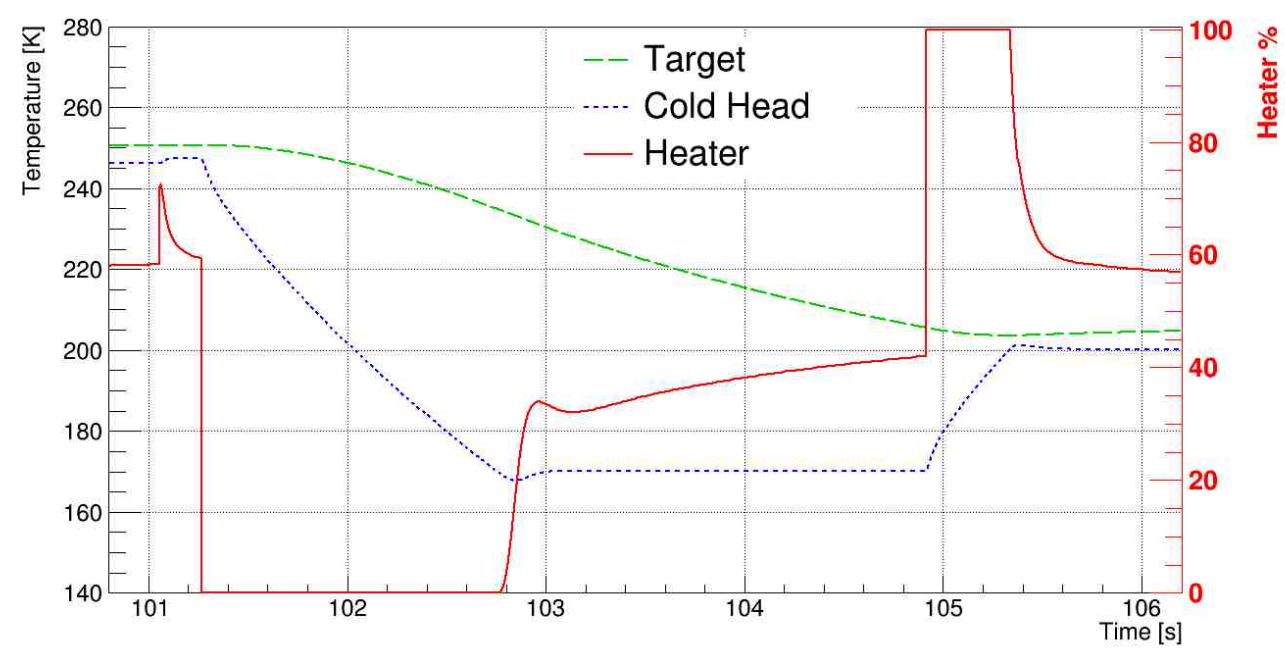

Figure 5. Cooldown step from $250 \mathrm{~K}$ to $205 \mathrm{~K}$. Temperatures of target and cold head are shown (left scale) together with the heater power (right scale) as a function of the elapsed time.

\subsection{The Ce:LaBr 3 X-ray detectors with PMT readout}

The study of muon transfer rate time dependence requires a high speed and high resolution X-ray detection system. The FAMU collaboration developed a fast detector system based on Ce:LaBr 31 1" scintillation crystals. Their high light output $(63,000$ photons $/ \mathrm{MeV}$ at $380 \mathrm{~nm})$ and fast pulse time decay (16 ns) allow for high counting rate, excellent energy and time resolution [14]. The high light output and the high absorption efficiency for X-rays (85\% at $300 \mathrm{keV}$ for 1" diameter crystal [15]) allow very good spectroscopic performances (less than 3\% of FWHM at $662 \mathrm{keV}[14,16]$ ) when coupled with high quantum efficiency last generation photomultipliers (PMTs).

To maximize the collection of X-rays produced by the decaying muonic atom cascade, a set of eight detectors was arranged as close as possible to the external target shell. Taking into consideration the volume density of muons stopped in gas and the isotropic X-ray emission, the detectors are arranged in star shaped mechanical support (figure 2). All detectors are placed $3 \mathrm{~cm}$ away from the internal target shell containing the gas mixture. Detectors cover a total area of $40.5 \mathrm{~cm}^{2}$ which correspond to $4.5 \mathrm{sr}$ of solid angle. Each detector unit is composed by a 1"x1" (diameter $\mathrm{x}$ thickness) Ce: $\mathrm{LaBr}_{3}$ crystal coupled with a compact PMT. Crystal, PMT and the electronics are encapsulated in a custom, black ABS 3D printed holder which also provides light tightness (see figure 6). The integrated detector is inserted in a $3 \times 3 \mathrm{~cm}^{2} 2 \mathrm{~mm}$ thick Aluminium profile.

Crystals with similar performances were selected from three manufacturers: Scitilion (China), REXON (USA) and Saint Gobain (France). The PMTs are Ultra BiAlcali (UBA) cathode photomultipliers R11265U-200 by Hamamatsu Photonics K.K. with a quantum efficiency in the Ce:LaBr 3 spectrum peak $(\sim 380 \mathrm{~nm})$ of $\approx 43 \%$ [17]. The PMT voltage divider has to deal with high instantaneous current density in presence of the pulsed muon beam and given the whole detector characteristics. A custom active voltage divider was designed to ensure the best performances of the system, both in timing and spectroscopy, see reference [17] for further details. 

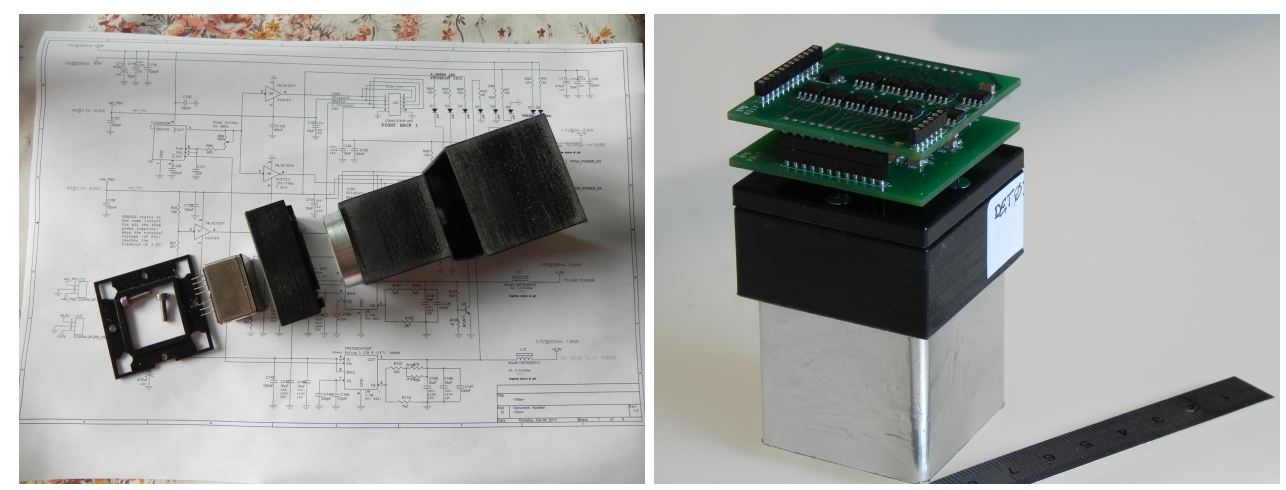

Figure 6. Left panel: disassembled detector: the circular $\mathrm{Ce}: \mathrm{LaBr}_{3}$ crystal and the squared PMT are visible. Right panel: assembled detector with part of the electronic visible, in particular the active voltage divider MOSFETs.

\subsection{Compact X-rays detectors with SiPM array readout}

Detectors more compact than Ce:LaBr3 crystals with PMT readout are needed to equip the remaining regions, as the ones under the target vessel, otherwise difficult to be instrumented due to lack of space. The choice was to use $1 / 2^{\prime \prime}$ crystals with a SiPM array readout, where the detector volume is only slightly bigger than the crystal dimensions. In the 2016 run, two 1/2" Ce:GAAG and four 1/2" Pr:LuAG detectors were used. In the 2017 and following runs, they were replaced by eight 1/2" Ce:LaBr 3 . The $1 / 2$ " crystals are read by a $4 \times 4$ array made of $3 \times 3 \mathrm{~mm}^{2} \mathrm{SiPM}$. The detector holder was realized with a 3D printer. Operating voltages are set according to manufacturer's specifications. Most of our results were obtained with Hamamatsu S13361 arrays, based on a TSV ("Through Silicon Via") technology. For Pr:LuAg with emission in the near UV (NUV) and $\mathrm{Ce}: \mathrm{LaBr}_{3}$ crystals SiPM arrays with a Silicone window were used to increase response at NUV wavelengths. The output from each pixel of the $4 \times 4 \mathrm{SiPM}$ array is summed up on a custom PCB. For more details see reference [18].

To correct for the temperature gain drift of the SiPM arrays, an online correction is implemented via a Nuclear Instruments NIPM12 digital controlled power supply, with a temperature feedback provided by a onboard temperature sensor.

\subsection{The HPGe X-ray detectors}

In all the runs of the FAMU experiment, four HPGe detectors complemented the $\mathrm{Ce}: \mathrm{LaBr}_{3}$ crystals for characteristic X-rays detection. Their main aim, covering only a small fraction of the solid angle, was to provide a high precision inter-calibration for the whole detection system, due to the better energy resolution of HPGe detectors as compared to $\mathrm{Ce}: \mathrm{LaBr}_{3}$ ones. ${ }^{2}$ They are also useful to identify contaminations in the gas and background sources.

The four HPGe detectors were two Ortec GEM-S, one Ortec GLP and one Ortec GMX detector. The GEM-S detectors have a semi-planar geometry, p-type with diameter $\times$ length $30 \mathrm{~mm} \times 20 \mathrm{~mm}$ and a $0.9 \mathrm{~mm}$ carbon window. The GLP detector has a planar geometry, n-type

\footnotetext{
${ }^{2} \mathrm{As}$ an example, at $122 \mathrm{keV}$ an Ortec GLP (GEM-S) detector has an energy resolution of $0.4(\%)(0.7 \%)$ (FWHM) to be compared with $8 \%$ from a typical $\mathrm{Ce}: \mathrm{LaBr}_{3}$ detector.
} 
with diameter $\times$ length $16 \mathrm{~mm} \times 10 \mathrm{~mm}$ and a $0.127 \mathrm{~mm}$ beryllium window. The Ortec GMX is instead a coaxial n-type detector with diameter $\times$ length $54.8 \mathrm{~mm} \times 49.8 \mathrm{~mm}$ and a $0.127 \mathrm{~mm}$ beryllium window.

HPGe detectors signals shaped through a preamplifier and a shaper were sent, after a splitter, both to a CAEN V1724 $100 \mathrm{MHz}$ FADC and a Ortec MCB Multichannel Analyzer for a fast online analysis, via the MAESTRO software [19]. For the four HPGe detectors Ortec 672 spectroscopic amplifiers were used. In addition, for one GEM-S HPGe detector also a fast Ortec 579 shaper was used. The shaping time was $2 \mu$ s and 200 ns, respectively for the Ortec 672 and the Ortec 579 modules.

Studies are under way to develop a faster pre-amplifier, with a $\leq 100 \mathrm{~ns}$ risetime, to be compared with a standard of $\sim 200 \mathrm{~ns}$

\subsection{Beam momentum tuning and detectors positioning}

The optimal disposition of the detectors depends on the distribution of the muon stop in the target. X-rays coming from inert material, especially during the arrival of the muon spill, must be minimized while maximizing the X-rays produced in the gaseous target. The muon stop in the apparatus depends on the amount and types of material along the beam line and on the momentum and spatial spread of the beam.

A Monte Carlo simulation has been used to determine the best configuration. The GEANT4 simulation toolkit [20] (version 4.10.01) has been used in conjunction with the Generic GEANT4 Simulation software ${ }^{3}$. The software provides a set of tools for signal hit readout and the possibility of a runtime plug-in detector geometry implementation using external shared libraries.

The FAMU GEANT4 simulation geometry reproduces the lead collimator, the target, the cryogenic vessel and detectors with their ancillary components, as insulating multi-layer foils, supports and inert materials. The simulation takes into account the geometrical physical dimensions and the materials used to construct the target.

The muon beam was simulated according to [21], i.e. with a circular shape of $4 \mathrm{~cm}$ diameter, divergence of $60 \mathrm{mrad}$ at the exit of the beam pipe and a momentum spread $\sigma_{p} / p \approx 4 \%$.

By varying the beam momentum in the simulation, it was possible to study the muon stop in the apparatus materials. Figure 7 shows the resulting fraction of events stopping in different materials as a function of the beam momentum. It may be noticed how the maximum muon stop in the gas almost matches the mininum stop in the Aluminium at about $57 \mathrm{MeV} / \mathrm{c}$ beam momentum. The muon stop in heavier elements, as Gold and Nickel, does not interfere with the transfer rate measurement since the muon capture by the nuclei happens faster than the thermalization and delayed phase of the muonic Hydrogen under study.

Once the muon beam momentum has been fixed, it is possible to study the spatial distribution of muon stop in the gas as shown in figure 8, top panel. The figure shows the ZX projection of stopping muons - the Z-axis being along the beamline. Most of the muons stop in the front part of the target, even if a fraction of them reach the rear, filling the whole target.

The fast Ce:LaBr 3 crystals read by PMTs were placed close to the target in the region of maximum stop of muons in the gas. They were placed inside two half circular crown supports,

${ }^{3}$ GGS v. 2.1, developed as a fork from the official simulation setup written for the GAMMA-400 experiment by the Trieste WiZard group and available via git: "git clone git://wizard.fi.infn.it/GGSSoftware.git". 


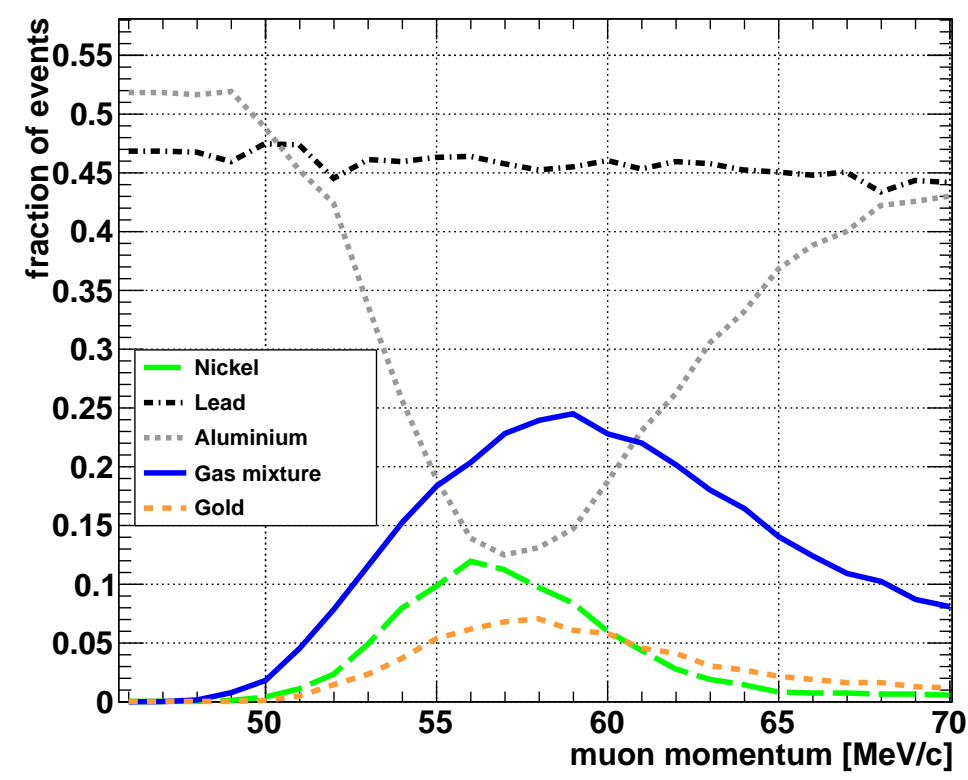

Figure 7. GEANT4 simulation of the muon stopping in the FAMU apparatus. The figure shows the fraction of events stopping in the gas (blue solid line), in the Aluminium walls (gray dotted line), in the Lead collimator (black dotted-solid line), in the coating (Gold yellow dotted line, Nickel green line) as function of muon beam momentum.

as shown in figure 8 middle panel. Bottom panel shows the distribution of muon stop inside the simulated target.

Other type of crystals read by SiPMT, in 2015 and 2016, were placed, thanks to their compactness, under the target inside a T-shaped 3D-printed support, figure 9 left panel. Since 2017, the Ce: $\mathrm{LaBr}_{3}$ crystals read by SiPMTs were instead positioned in the front part of the target using two half circular crown supports which completed the ones of $\mathrm{Ce}: \mathrm{LaBr}_{3}$ read by PMTs, as shown in figure 9 on the right.

HPGes detectors, due to their longer shaping time, are more sensitive to X-rays pile-up. For this reason their position was farther away from the target respect to the other detectors, usually pointing to the rear part of the apparatus. Figure 10 shows the position of the HPGe detectors during the 2016 acquisition. Also shown is the hodoscope placed in front of the target.

\section{Raw data handling}

The data processing from online to offline analysis is outlined in the following.

Signals (64 in all) from each hodoscope detector are digitized by two CAEN V1742 digitizer in VME standard, while signals from the crystal detectors (eight with PMT readout and eight with SiPM array readout) are digitized by V1730 or DT5730 digitizers. The signals from the four HPGe are instead digitized by a CAEN $100 \mathrm{MHz}$ V1724 digitizer. The V1742 modules are read via a CAEN V2718 VME-PCI interface, while instead the V1730, DT5730 and V1724 digitizers are read directly with an optical fiber via a CAEN A3828 PCI card, to have a faster transfer rate. The 

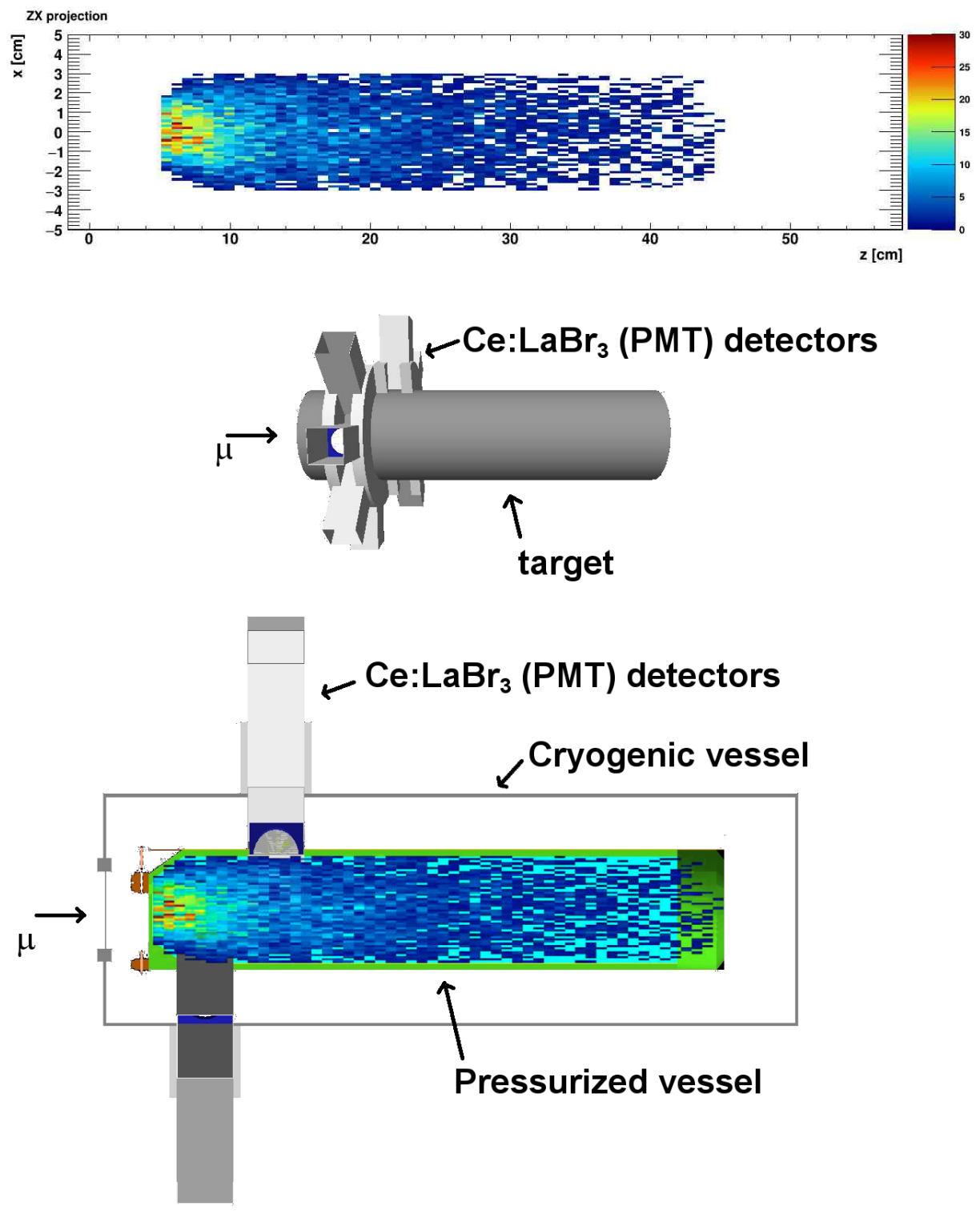

Figure 8. Top panel: distribution of $57 \mathrm{MeV} / \mathrm{c}$ muons stopping in the target. Middle panel: drawing from the GEANT4 simulation of the target and the Ce:LaBr 3 crystals read by PMT. Bottom panel: section of the previous drawing with the muon stop distribution overimposed.

beamline gives an adjustable pre-trigger signal, typically $500 \mathrm{~ns}$ before the center of the time at which the first muon spills reaches the target. Muons are bunched in two spills, with $70 \mathrm{~ns}$ width, at $320 \mathrm{~ns}$ distance. Thus the chosen windows of $5 \mu$ s for hodoscopes, $10 \mu$ s for $\mathrm{Ce}: \mathrm{LaBr}_{3}$ detectors and $20 \mu \mathrm{s}$ for HPGe detectors were enough for containing the full signal waveform. The waveforms were sampled every $2 \mathrm{~ns}$ for the hodoscopes and the crystal detectors. From signal waveforms the most complete set of information may be deduced, such as peak position, timing, etc. Runs of 10000 events were recorded on disk by a custom made data acquisition software and stored as PAW ntuples. These ntuples are converted later to ROOT files for offline analysis. 

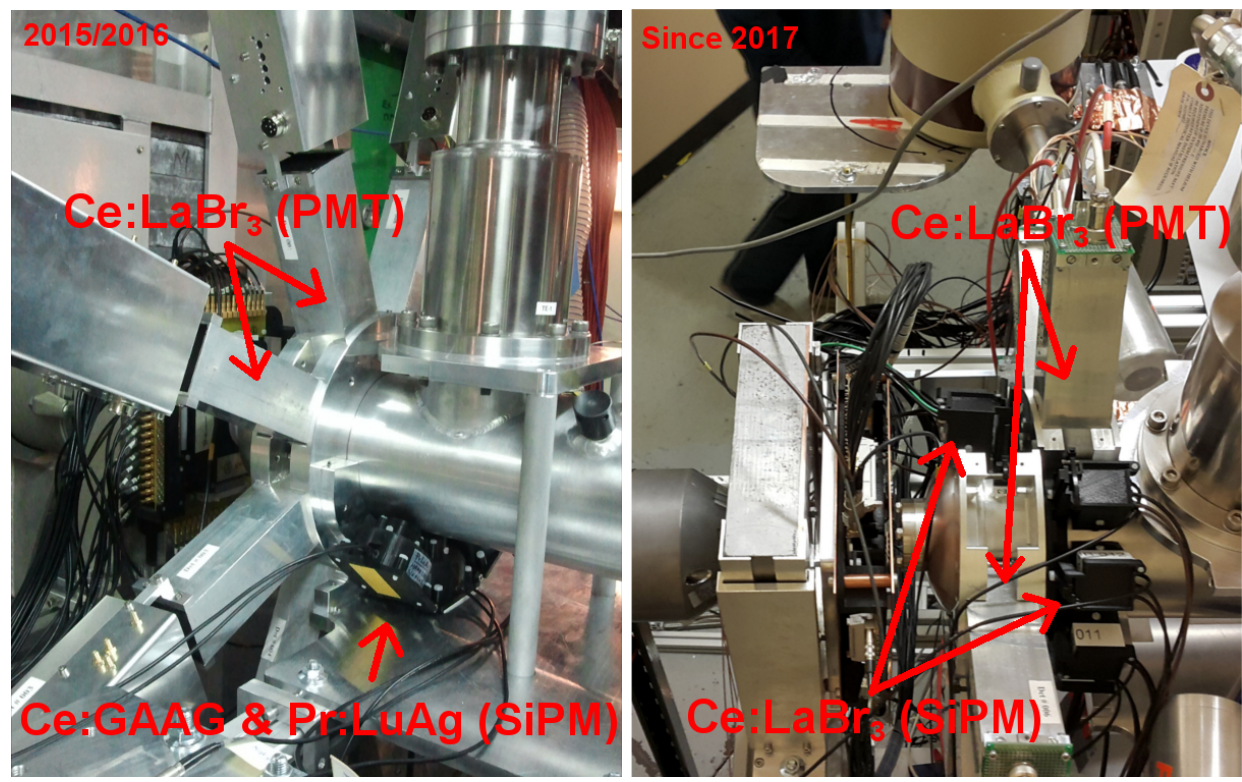

Figure 9. Left panel: positioning of the T-shaped support structure with crystals read by SiPM (2015 and 2016 data taking). Right panel: positioning of the $\mathrm{Ce}: \mathrm{LaBr}_{3}$ crystal detectors since 2017.

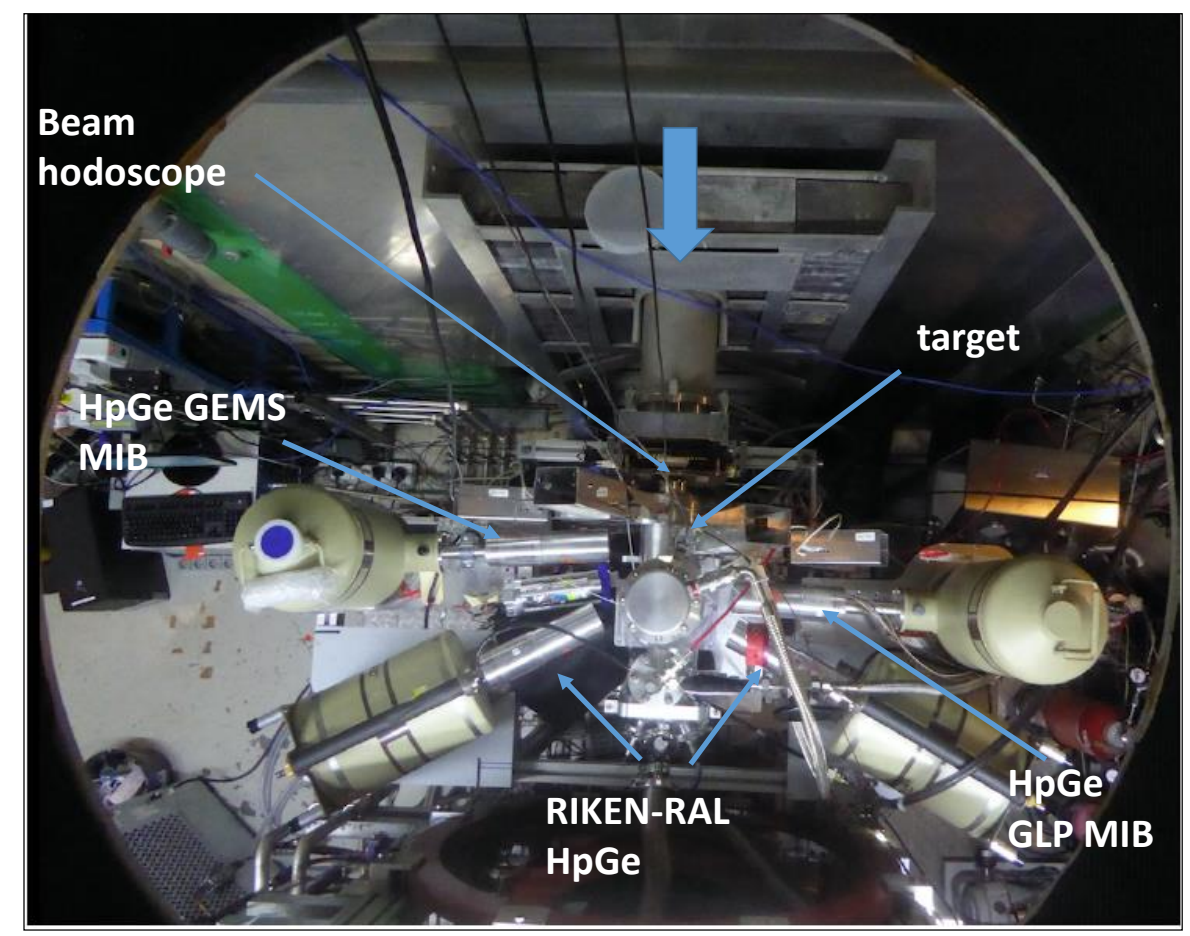

Figure 10. Picture of the FAMU layout seen from the top. The position of the HPGe detectors and the $1 \mathrm{~mm}$ pitch beam hodoscope are shown.

Further details on the online data acquisition system are shown in reference [22]. 

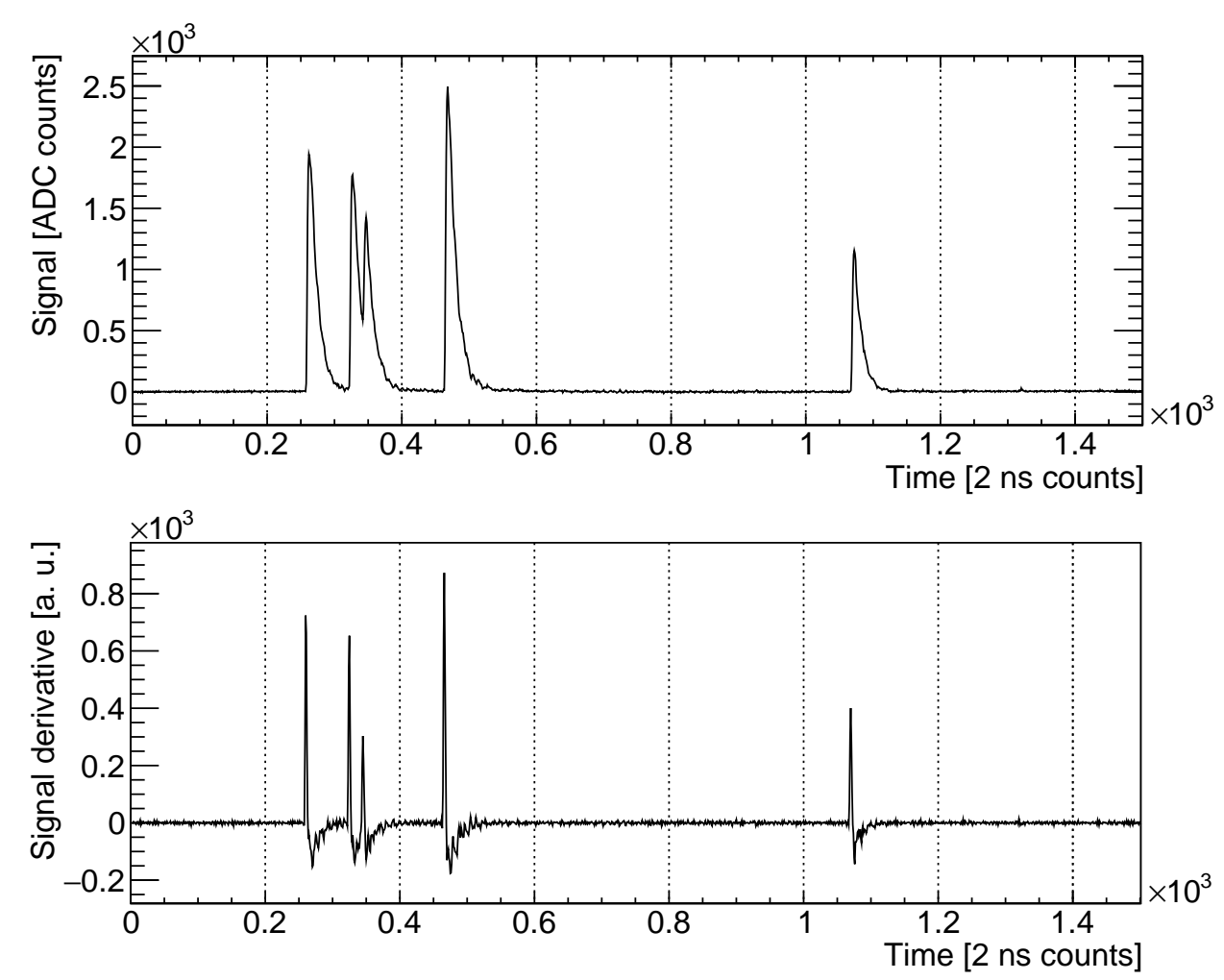

Figure 11. Top panel: unpacked waveforms for a typical $\mathrm{Ce}: \mathrm{LaBr}_{3}$ detector channel. Bottom panel: computed signal derivative. To each wavefront corresponds a large spike in the derivative. One count corresponds to two nanoseconds.

\subsection{Structure of offline data processing}

Data processing sofware is written in C++ language, and makes use of the ROOT toolkit [23]. Acquired raw data are in PAW ntuples format. They are converted in ROOTples containing the detectors waveforms packed in data trees.

Raw data (PAW and ROOT files) are automatically transferred from the local DAQ storage disk to a remote repository via a Grid-FTP protocol. The main data processing program reads the raw files and retrieves the observables needed in the analysis.

The data processing software has two main operating modes. The first one is very fast (the processing time is smaller than acquisition time) and requires very little CPU power. This mode ("quick-look") is used to monitor the data flow during the acquisition procedure and it is used to produce data quality plots. The second mode gives more accurate results for the data analysis but it is significantly slower (the processing time is about ten times greater than the acquisition time), since fitting algorithms are involved.

The data processing program treats the output from the various detectors in different ways that depend on the waveform shape and detector characteristics.

Hodoscopes are used to study the muon beam shape and intensity. Their signal is different from X-rays detectors and the processing program has a dedicate routine to process their waveforms. Both in the "quick-look" and in the "full analysis" modes, the software reads the raw ROOT input 
file, measures the total energy deposit, above a fixed threshold, of each of the fiber strips and saves the data in a new ROOT file.

$\mathrm{X}$-rays detectors (HpGe, $\mathrm{Ce}: \mathrm{LaBr}_{3}$ and other crystals) have most of the core routines in common. For all the detectors and in both the processing modes, the program flow is the following:

- for each detector the waveforms are unpacked event by event, as shown in the top panel of figure 11;

- a numerical derivative of the waveform is computed with a finite difference method using a five-point stencil in one dimension. In addition, resulting sharp edge due to signal saturation are smoothed by recognizing flat top of the saturated waveforms. An example of signal derivative is shown in figure 11, bottom panel. A fixed threshold is used to find the X-rays signals. The software identifies the starting point of the signal window when the derivative rises above the threshold. The approximate position of the maximum is given by the derivative zero crossing. The threshold value was set empirically in order to minimize the number of fake triggers, due to the intrinsic baseline noise, and maximize the number of detected Xrays. The determined threshold value on the derivative depends on the detector and, e.g. for $\mathrm{Ce}: \mathrm{LaBr} 3$ read by PMTs, corresponds to about $50 \mathrm{keV}$ X-rays signals.

- the energy of each X-ray signal is reconstructed together with its arrival time;

- the amplitude of each signal and its starting time is saved in the output data structure, together with other informations (number of the detector, and housekeeping informations as the target temperature and the absolute linux time);

- the output data structure is saved in an output ROOT file.

The same program checks the data for signal saturation (mostly due to the digitization electronics) and saves the information in the same output file. The method to determine energy and starting time of each X-ray depends on the detector and on the analysis mode.

The fastest detectors are the $\mathrm{Ce}: \mathrm{LaBr}_{3}$ crystals read by PMTs. They have been used to study the physics of transfer rate of the muon from muonic Hydrogen to other elements in the 2016 data taking. The energy of each signal is reconstructed with two different algorithms, depending on the operating mode. The first one, used in the "quick-look" processing, determines the difference between the baseline and the maximum of the waveform in each identified signal window. This method is fast and works at its best with a low fluence of X-rays, but it is able to resolve with a low efficiency the overlapping signals (pile-up). A better approach, used in the analysis mode, has been implemented in which each signal is fitted with a function representing the signal shape. In case of pile-up a sum of signal-shape functions is used in order to measure correctly the energy of each $\mathrm{X}$-ray. As an example, in figure 12 the red line represents the total fit function, the blue lines the single components due to each X-ray, while the black points are the data to be fitted. The normalized $\chi^{2}$ of the fit, the degrees of freedom and exit fit status are stored together with the data. This pile-up reduction by the multiple fitting algorithm is the most compelling task of the data processing. A fine tuning of input parameters and the excellent stability of the detectors waveforms allowed a reliable reconstruction of piled up events. The software was tested using simulated waveforms 


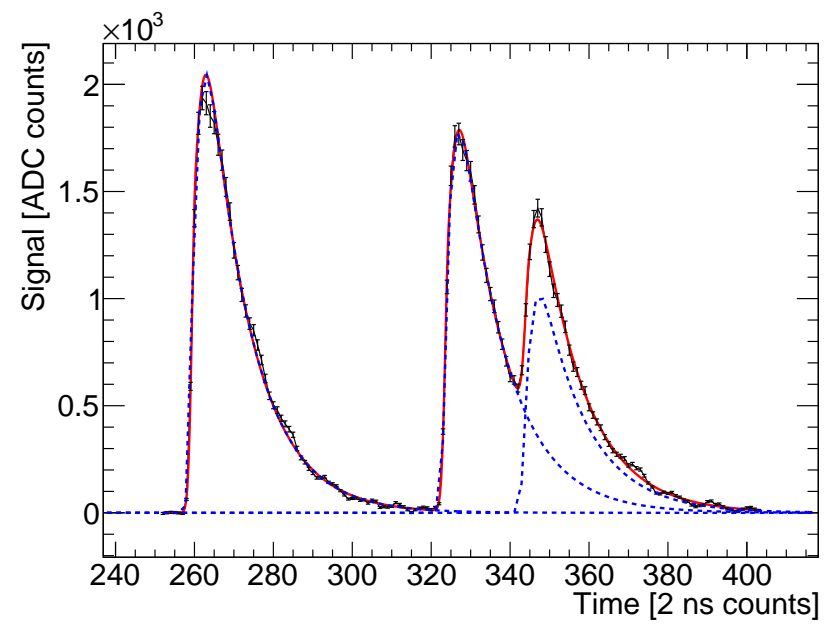

Figure 12. Example of multiple overlapping signals. Black points represent the data waveform, red line is a fit of the data using three signal shapes shown as blue lines.

(with included simulated electronic noise). Placing constraints on the resulting $\chi^{2}$ of the fit and on the distance between two neighbour signals, an efficiency greater than $90 \%$ was obtained for X-rays detected in the delayed phase, where the transfer rate is going to be measured. Efficiency drops to about $50 \%$ when the time distance between two X-rays signals is shorter than about $30 \mathrm{~ns}$. In this situation, which is present only during the prompt arrival of muons in the target, only the first signal energy is correctly reconstructed. The "perfect pile-up", i.e. the situation in which the software is not able to distinguish two signals, starts at a separation of less than $15 \mathrm{~ns}$ between signals. A Monte Carlo simulation proved that this phenomenon is negligible in the delayed phase.

The HPGe detectors amplified signals are usually too slow to detect many X-rays during the same trigger. Hence, the processing software for both the "quick-look" and the "full analysis" modes scan the whole waveform and save the maximum of the signal amplitude and its time, one $\mathrm{X}$-ray per each trigger (the information about signal time windows is not used in this case).

HPGe pre-amplified and fast-amplified signals and other fast X-ray detectors signals (Ce: $\mathrm{LaBr}_{3}$ and other type of crystals read by SiPMT) are processed using the same procedure. The quicklook mode uses the same maximum minus mininum algorithm described for germanium amplified signals. The analysis mode, instead, look for signal maximum in each signal window, the same algorithm described for the quick-look mode of $\mathrm{Ce}: \mathrm{LaBr}_{3}$ read by PMTs.

Finally, data for each detector are calibrated offline in order to convert from arbitrary digitizer units to $\mathrm{keV}$. This is usually done by processing the source calibration data taking and by knowing the characteristic X-rays nominal peak positions of the source. Calibration parameters are saved in a MySQL database and used in the following data analysis.

\section{Experimental operations and performances}

The operations of the various elements of the FAMU experiment at RIKEN-RAL will be described in detail in the following. 


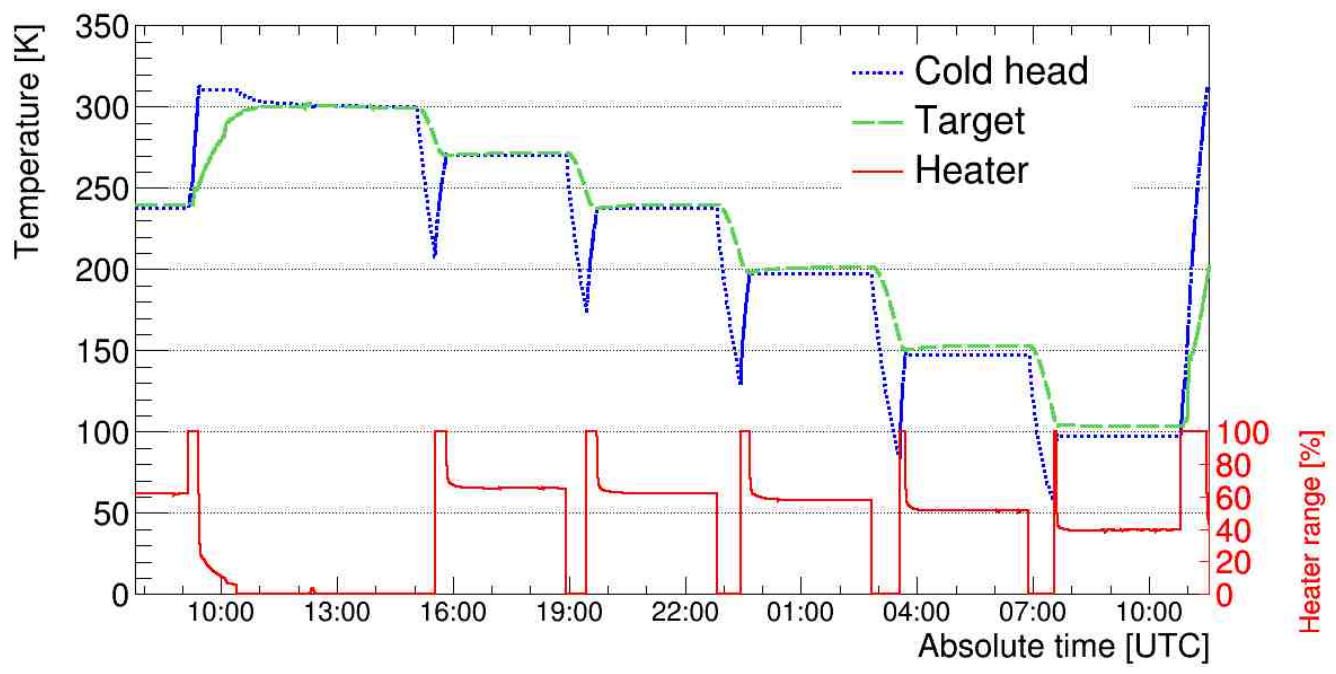

Figure 13. Example of a temperature cycle performed on the beam with the target in 2016. Blue line cold head sensor, green line target sensor, magenta line heater power percentage.

\subsection{Target operations}

A first set of measurements on the RIKEN-RAL muon beam, with the FAMU high pressure gas Hydrogen target and detectors allowed us to successfully validate the target system.

The target filling procedure requires that first a turbo-molecular vacuum pump is used to clean the gas target. On the beam setup at RAL, the target at $300 \mathrm{~K}$ temperature is evacuated until the pressure is measured to be as low as $5 \times 10^{-6}$ bar. Then three cycles of gas filling (at 10 bar) and flushing with pure Hydrogen are performed. After having re-created the vacuum with the turbo-molecular pump, the gas mixture needed for the measurement is injected in the target.

At any injection the measured temperature increases up to 10 degrees $\mathrm{K}$, due to the heating of the gas when crossing valves and tubes. This effect was studied carefully in 2017 by measuring the pressure and temperature of the target during and after the filling. For example, the target filled at 40 bar starting at $300 \mathrm{~K}$ increases its temperature up to about $310 \mathrm{~K}$; when the temperature is brought back to $300 \mathrm{~K}$ the measured pressure is of 39 bar. This means that a drop in the pressure of about $2.5 \%$ is observed respect to the nominal filling pressure. In 2016 data taking, the pressure was measured only at filling time, so the related data analysis has to include the $2.5 \%$ correction in the pressure measurement.

Once filled and thermalized, the target is used for the measurements at different temperatures. An example of temperature cycle is shown in figure 13, during the February 2016 data taking with $\mathrm{H}_{2}+\mathrm{O}_{2}(0.3 \%)$ gas mixture. After the filling, at 10:00 a.m., three hours of data are acquired. The blue line represents the cold head temperature measurement. It varies very fast depending on the power induced by the thermo-resistance heater. The percentage of power is shown as a magenta line in the same figure. The temperature of the target, green line, varies much more smoothly, since the heat is brought to the target with copper connections. As described in section 2.2, to make the transition between different temperatures faster, the cold head was brought to a lower temperature than the target one and only when the target temperature was approaching the desired value the cold 

was dead in this particular run, taken in 2016, this was due to a broken signal cable.
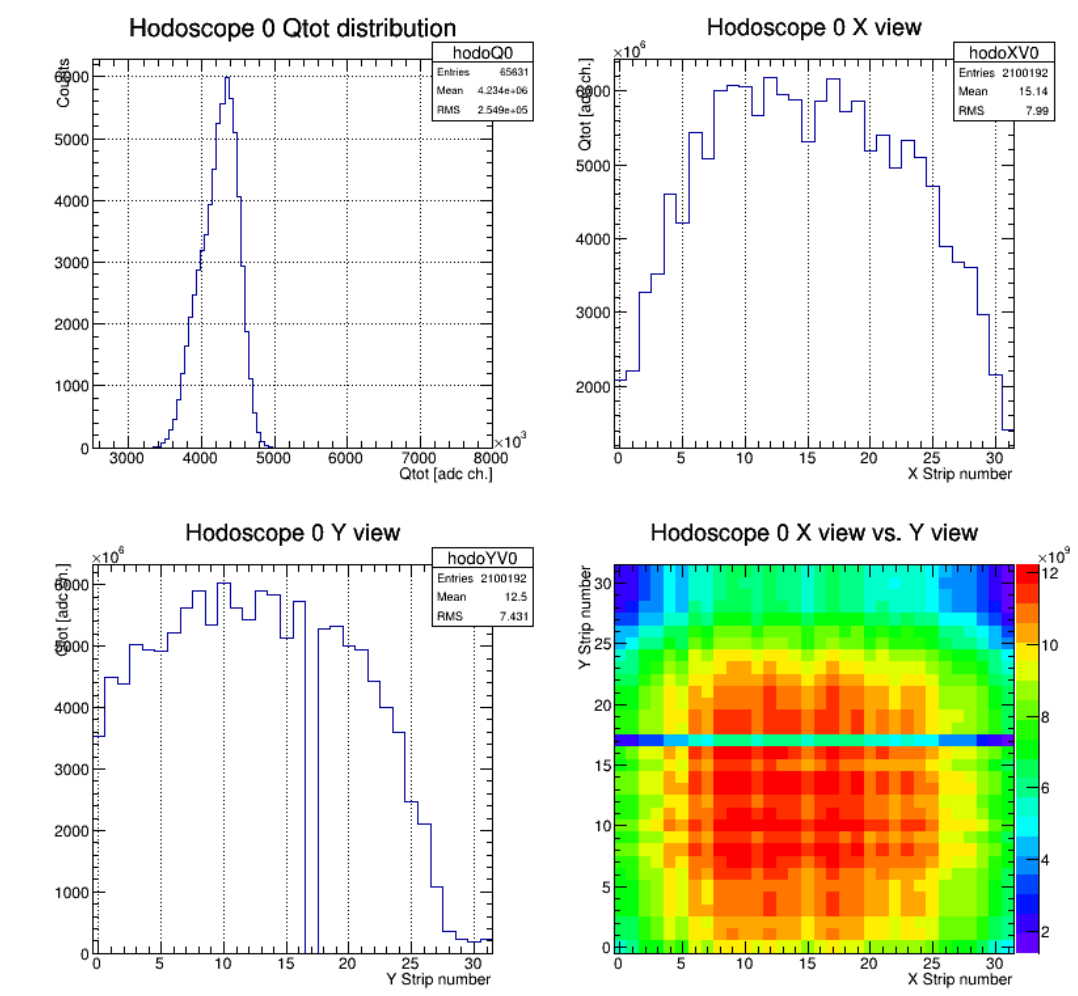

Figure 14. $\mathrm{X} / \mathrm{Y}$ beam profile at RIKEN-RAL for a $57 \mathrm{MeV} / \mathrm{c}$ run. The accumulated total charge is also shown.

head was heated again. This can be clearly seen in the figure at about 16:00, where the cold head temperature goes down to about $200 \mathrm{~K}$ while the target temperature is changes from 300 to $273 \mathrm{~K}$. Six steps of three hours each were taken at the target temperatures of 300, 273, 240, 200, 150, and $100 \mathrm{~K}$. Once the cycle ends, the target is heated to $300 \mathrm{~K}$ to clean up and flushing and to start a new cycle with a different gas mixture.

\subsection{Beam characterisation with the $1 \mathrm{~mm}$ pitch hodoscope}

The permanently installed $1 \mathrm{~mm}$ pitch beam hodoscope has been used at RIKEN-RAL to control and optimize the beam steering inside the target. The signal waveform for each channel was integrated, after subtracting the baseline, to provide informations for an X/Y occupancy plot. The X/Y beam profile for a typical run is shown in figure 14. The total charge collected on the beam hodoscope $\left(Q_{t o t}\right)$ is also shown and may be used to monitor the beam intensity. A channel of the hodoscope

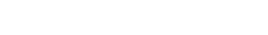

Making use of laboratory data taken with impinging cosmic rays, and using tabulated $\mathrm{dE} / \mathrm{dx}$ data from PDG [24], it was possible to estimate from the total charge collected $\left(Q_{t o t}\right)$ the number of beam muons. The spill rate (nominally $50 \mathrm{~Hz}$ ) was estimated from data itself and was $\sim 30 \mathrm{~Hz}$, as only three out of four spills reached the target in these runs. An example of the muon rate per second is shown in figure 15 for a momentum scan taken in 2018.

Results are compatible with previous results, reported in [6]. 


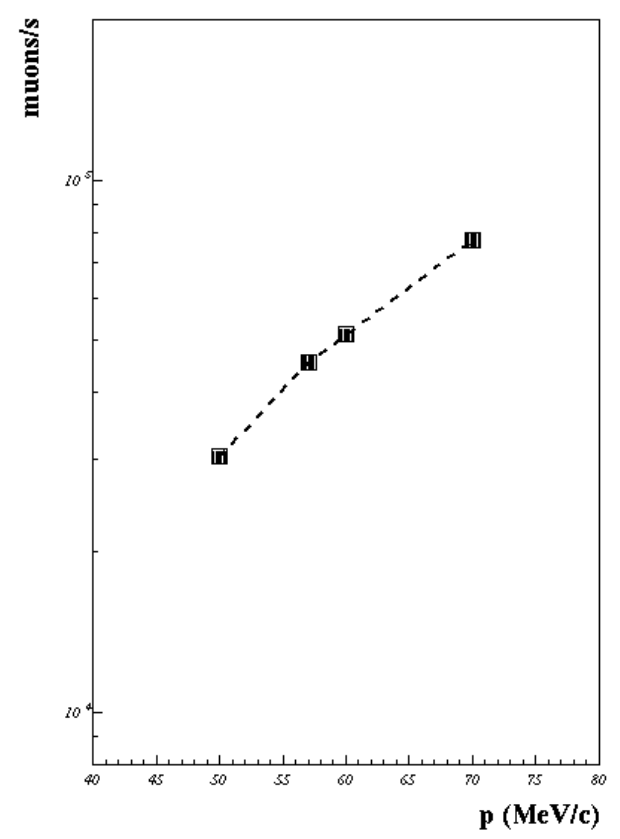

Figure 15. Muons per second at the target window, as measured in a scan during 2018 data taking.

\subsection{Detection of characteristic X-rays with $\mathrm{Ce}: \mathrm{LaBr}_{3}$ with $\mathrm{PMT}$ readout}

Due to the high rate of events, the minimum pile-up condition is preferred to the highest possible energy resolution.

The typical acquisition time window is $10 \mu$ s in which the PMT output signal is digitized at $500 \mathrm{Ms} / \mathrm{s}$ ( 2 ns time step). The whole window is divided in three zones (figure 16):

- pre-trigger, a small low counting zone, right before the arrival of muons [0 ns-400 ns];

- prompt phase, where muons arrive from the accelerator beam pipe and interacting with the target produce most X-rays events [400 ns-1000 ns];

- delayed region, the region of interest for the FAMU experiment. All the muonic X-rays emitted by transfer phenomena are produced here [1000 ns-10 $\mu \mathrm{s}]$.

At the muon beam arrival (double pulse shape), an average number of $4 \mathrm{X}$-rays are detected in $1 \mu$ s time slot, and up to $10 \mathrm{X}$-rays are detected in $10 \mu \mathrm{s}$ time window (see figure 11).

The detector signal for a typical X-ray has a peaking time of $\sim 12 \mathrm{~ns}$ and an exponential decay constant of $\sim 16 \mathrm{~ns}$. The PMT gain is set to fit the data acquisition dynamic range with maximum energy of about $1 \mathrm{MeV}$. A custom algorithm was developed to identify each single pulse in the full acquisition window, even in case of pile up (see section 3.1 for details). Energy and time of arrival for each X-ray event are stored. The algorithm is capable to resolve pile-up events down to $15 \mathrm{~ns}$ of peak separation. From $15 \mathrm{~ns}$ to $30 \mathrm{~ns}$ it has an efficiency of $\sim 50 \%$. It reaches a $90 \%$ efficiency after $30 \mathrm{~ns}$ of separation. This allows to record the largest number of events (increased statistic) losing only a small fraction of events due to pile-up. Given priority to the minimization 


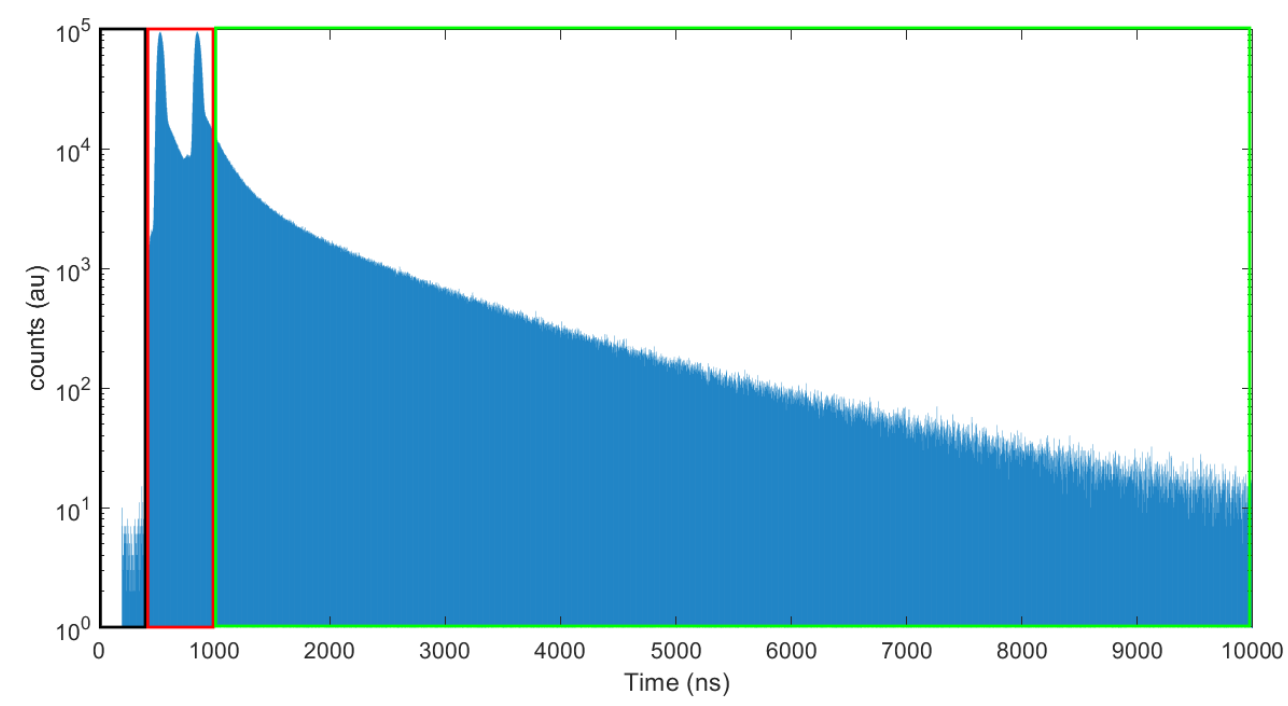

Figure 16. Time spectra from $\mathrm{Ce}: \mathrm{LaBr}_{3}$ with PMT readout. Three regions are indicated: pre-trigger in black, prompt phase in red and delayed region in green.

Table 2. Obtained FWHM resolution of Ce: $\mathrm{LaBr}_{3}$ crystals with PMT readout as reported in literature and measured from calibration runs with ${ }^{57} \mathrm{Co}$ and ${ }^{137} \mathrm{Cs}$ sources.

\begin{tabular}{|l|c|c|}
\hline Energy & FWHM from ref. [16] & measured FWHM \\
\hline $122 \mathrm{keV}$ & $7.4 \%$ & $8.8 \%$ \\
\hline $662 \mathrm{keV}$ & $2.8 \%$ & $3.5 \%$ \\
\hline
\end{tabular}

of pile-up the energy resolution is affected. In fact, this is slightly worse as respect to the achieved ones in literature (see table 2 for further details). To evaluate the detector performances during the data taking (in high instantaneous X-ray flux conditions) we measured the energy resolution of the $133 \mathrm{keV}$ muon Oxygen $K_{\alpha}$ line acquired right after the prompt phase (1000 ns-10 $\mu \mathrm{s}$ ). Theoretically, energy resolution varies in respect with energy, following an inverse quadratic rule, so it is possible to extrapolate the expected resolution at any energy from calibration data. In particular, using the $8.8 \%$ as FWHM resolution for the $122 \mathrm{keV}{ }^{57} \mathrm{Co}$ source (see table 2) we expect $8.4 \%$ at $133 \mathrm{keV}$. Experimentally we found $8.5 \%$ (figure 17) that is compatible within the total systematics uncertainties. This demonstrates the good detector performance in both high and low rate conditions.

In conclusion, the good detectors performances, combined with a proper analysis fitting algorithm have permitted to achieve both good spectral and timing performances with excellent detection efficiency. These performances fit well with the experimental requirements: study the X-rays spectrum time evolution from the prompt region to the delayed one. The time evolution of the $\mathrm{X}$-rays emission of the Hydrogen (99.7\%) Oxygen (0.3\%) mixture is shown in figure 18. It is possible to notice that the $133 \mathrm{keV} K_{\alpha}$ and $158 \mathrm{keV}+167 \mathrm{keV}$ (respectively $K_{\beta}$ and $K_{\gamma}$ ) muonic Oxygen lines are emitted for few hundreds of nanosecond after the prompt ones. During the first 


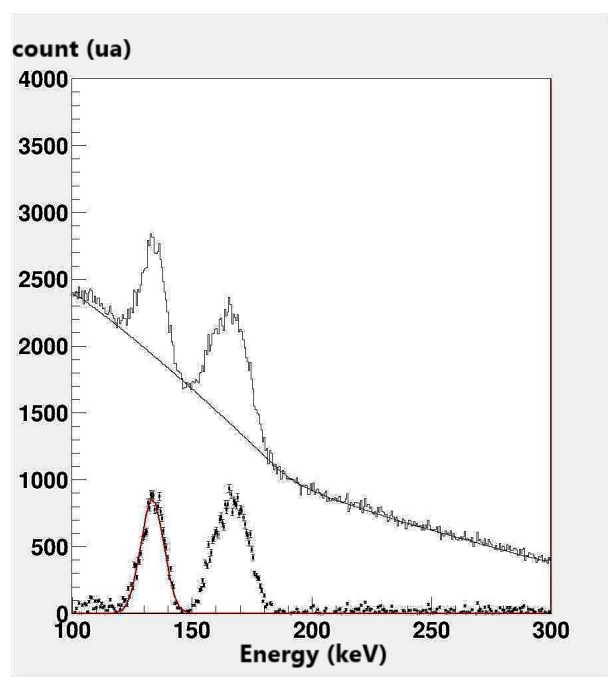

Figure 17. Measured delayed spectrum with a $\mathrm{H}_{2}+\mathrm{O}_{2}(0,3 \%)$ gas mixture in the target. Muonic Oxygen X-rays: $133 \mathrm{keV} \mathrm{K}_{\alpha}, 158 \mathrm{keV} \mathrm{K}_{\beta}$ and $167 \mathrm{keV} \mathrm{K}_{\gamma}$ lines, with background (top) and background subtracted (bottom). The energy resolution for the $133 \mathrm{keV}$ line is $8.5 \%$.
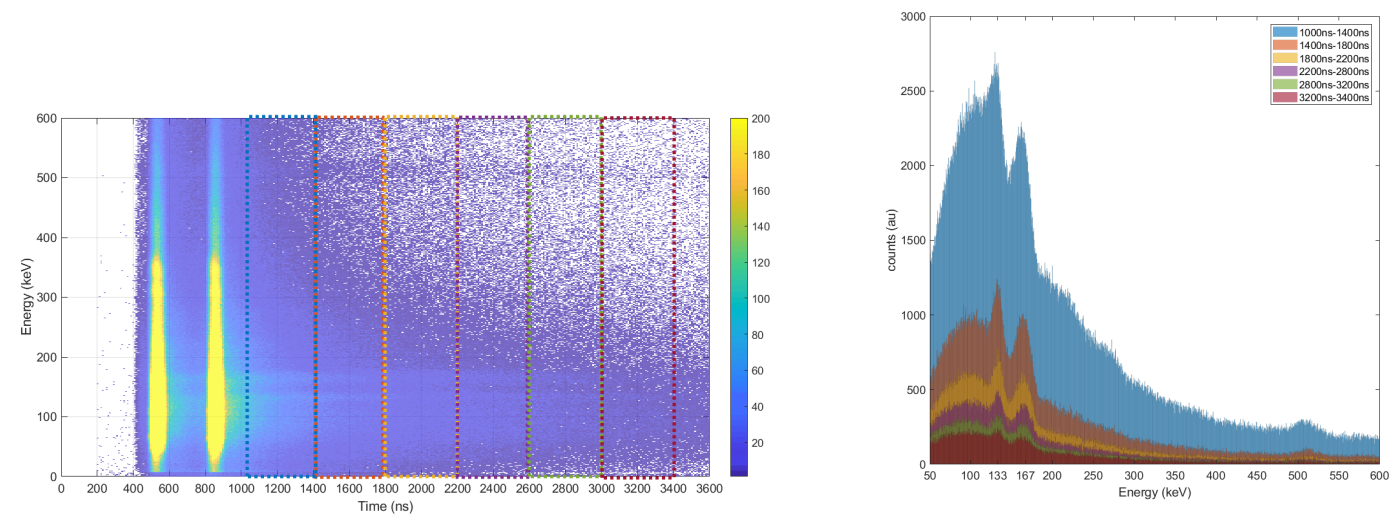

Figure 18. Left panel: $\mathrm{X}$-ray time evolution spectrum of the $\mathrm{H}_{2}-0.3 \% \mathrm{O}_{2}$ mixture with a muons momentum of $\mathrm{P}=57 \mathrm{MeV} / \mathrm{c}$. Right panel: $400 \mathrm{~ns}$ integrated time cut spectra right after the prompt phase (from $1000 \mathrm{ns).}$ All the time cut selections are shown in the top panel.

phase of the FAMU experiment, these detectors allowed the high precision studies on the transfer rate from muonic Hydrogen to Oxygen $[8,9]$.

\subsection{Detection of characteristic X-rays with crystals with SiPM array readout}

Pr:LuAG and Ce:GAAG crystals read by SiPM arrays (see section 2.4 for details) were placed under the cryogenic target in the 2016 data taking as additional X-rays detectors. They were initially calibrated in situ with a ${ }^{57} \mathrm{Co}$ source and a source that was an admixture of ${ }^{241} \mathrm{Am},{ }^{90} \mathrm{Sr}$ and ${ }^{137} \mathrm{Cs}$. As an example, at the ${ }^{137} \mathrm{Cs}$ peak for a Ce:GAAG detector the energy resolution (FWHM) was $\sim 6.9 \%$. Due to their poor positioning in the detector layout, in the 2016 data taking it was not possible to detect Oxygen X-lines from delayed events, even if the detectors were able to see the 
Table 3. Obtained FWHM resolution of $\mathrm{Ce}: \mathrm{LaBr}_{3}$ crystals with SiPM arrays as measured from calibration runs with ${ }^{57} \mathrm{Co}$ and ${ }^{137} \mathrm{Cs}$ sources obtained in situ at RAL.

\begin{tabular}{|l|c|c|c|}
\hline used array & $\begin{array}{c}59 \mathrm{keV} \\
\text { FWHM }(\%)\end{array}$ & $\begin{array}{c}122 \mathrm{keV} \\
\text { FWHM }(\%)\end{array}$ & $\begin{array}{c}662 \mathrm{keV} \\
\text { FWHM }(\%)\end{array}$ \\
\hline Hamamatsu 13361-AS (6 pcs) & $14.5 \pm 1.6$ & $9.6 \pm 1.4$ & $5.0 \pm 0.9$ \\
Hamamatsu 13361-AE (2 pcs) & $20.1 \pm 1.1$ & $13.4 \pm 3.1$ & $5.1 \pm 0.4$ \\
Advansid ASD-P-4-TD (1 pcs) & 20 & 11.8 & 5.5 \\
\hline
\end{tabular}

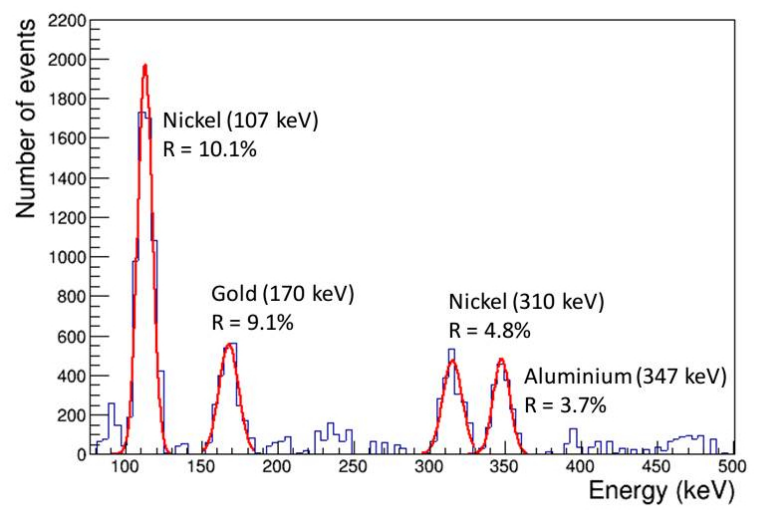

Figure 19. Spectrum with a pure Hydrogen filling of the target, as seen from one $\mathrm{Ce}: \mathrm{LaBr}_{3}$ detector, equipped with a Hamamatsu S13361-AS SiPM array. Background has been subtracted.

two beam pulses structure inside a spill (see reference [25] for further details).

In the 2017 run, the previous 1/2" crystals were replaced by $1 / 2$ " Ce:LaBr 3 crystals, to improve the energy resolution. The general detector's structure was left unchanged. In the following no attempt to correct SiPM arrays' gain for temperature excursions was done. Results with the calibration sources in situ are reported in table 3. They are compatible, within errors, with the expected $\frac{1}{\sqrt{E}}$ behaviour.

Results from calibration in situ are a little worse than the ones obtained in laboratory. This may be due to the presence of environmental electronic noise, effects due to temperature excursions, etc.

Different readout arrays were used, including Hamamatsu S13361 SiPM arrays, made with a TSV technology and Silicone (-AS) or epoxy (-AE) windows and Advansid SiPM arrays.

Using a target filled with pure Hydrogen (background) the results of figure 19 were obtained. $\mathrm{X}$-rays lines from materials present in the target, such as Nickel, Copper and Aluminium are easily recognized.

The detectors performance during data taking were then studied with delayed events (time larger than $2600 \mathrm{~ns}$ ) for a $\mathrm{H}_{2}+\mathrm{O}_{2}(0.3 \%)$ mixture in the 2017 run. Unfortunately, the used gas has been delivered by the supplier with an heavy contamination of $N_{2}$ and thus X-rays lines from both Nitrogen and Oxygen were present. Nevertheless, a spectral analysis was possible. For example a peak consistent with the $K_{\alpha}$ line from Nitrogen at $104.2 \mathrm{keV}$ is reconstructed with a FWHM=11.3 $\mathrm{keV}$, i.e. a resolution of $10.8 \%$.

These preliminary results in a situation of a heavily polluted gas mixture, make us confident that 
compact 1/2" Ce: $\mathrm{LaBr}_{3}$ crystals with SiPM array readout may be useful to instrument regions of the detector of difficult access. As data were taken along periods with relevant temperature excursions, the online correction of SiPM arrays' drift of gain with temperature will help to improve energy resolution up to the 4\% level (FWHM) obtained in laboratory tests in controlled environment [18].

\subsection{Detection of characteristic X-rays with HPGe detectors}

The signal of HPGe detectors, after amplification and shaping, is characterized by a sharp risetime $\sim 100-300 \mathrm{~ns}$ and a long tail $\sim 100-150 \mu \mathrm{s}$. A common limitation for the shaped signal in a high multiplicity environment is the pile-up effect, where saturation may show up. figure 20 shows for a run taken with the $\mathrm{H}_{2}+\mathrm{O}_{2}(0.3 \%)$ target two examples of signals from the GEM-S HPGe detector after the pre-amplification stage only, the amplification stage with a standard Ortec 672 spectroscopy amplifier (slow amplification, shaping time $2 \mu \mathrm{s}$ ) and the amplification stage with a fast Ortec 579 amplifier (shaping time $\sim 200 \mathrm{~ns}$ ). Two different situations are illustrated: in the top panels a pile-up event is shown, while in the bottom panels a saturated event is shown. It can be seen that the use of a fast amplifier mitigates the presence of pile-up events.
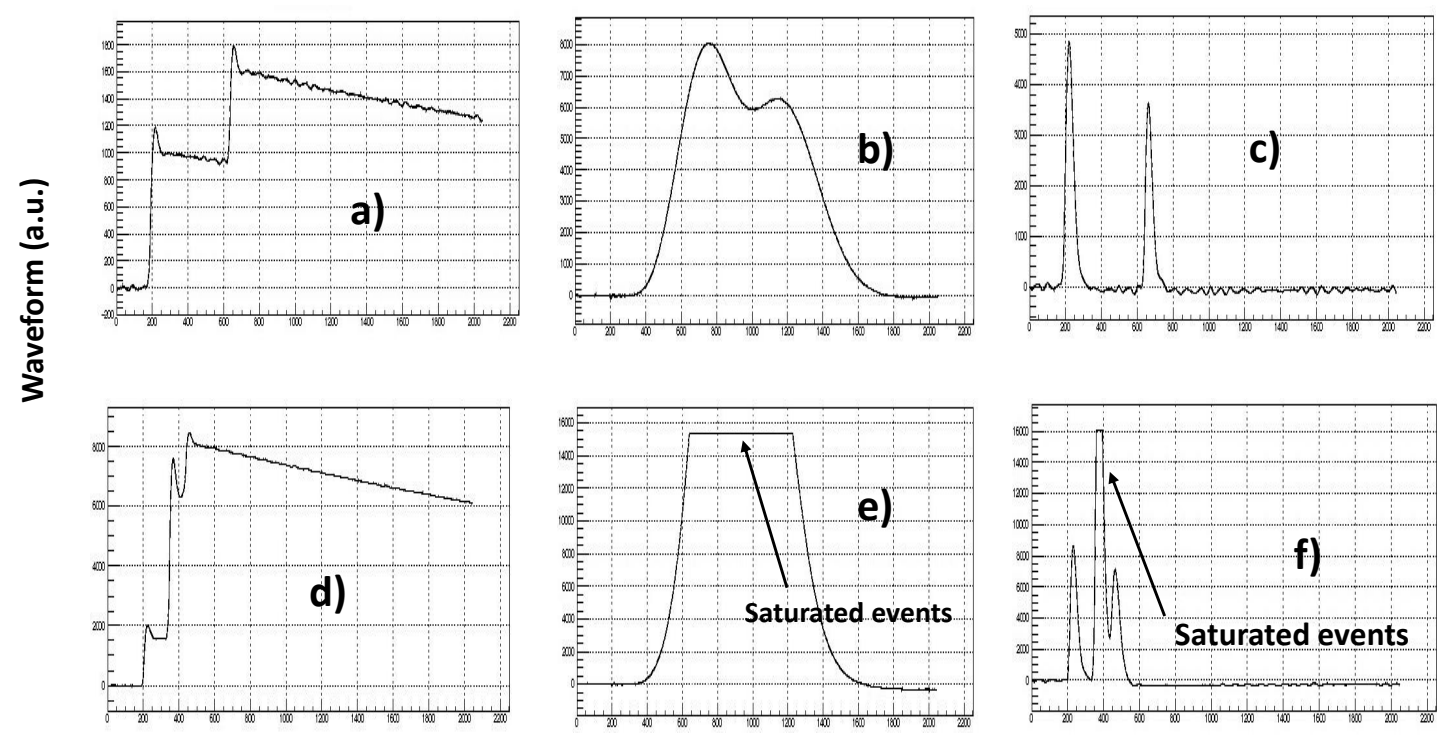

time (ns)

Figure 20. (a),(d) pre-amplified signals for a HPGe GEM-S detector. (b),(c) amplified signals with an Ortec 672 spectroscopy amplifier. (c),(f) amplified signals with a fast Ortec 579 detector.

The capability of HPGe detectors for intercalibration of the full apparatus is demonstrated in figure 21 for the HPGe GEM-S detector, with the $\mathrm{H}_{2}+\mathrm{O}_{2}(0.3 \%)$ target, where the energy spectrum from fast amplified signals with an Ortec 579 are shown. Characteristic X-rays lines in the region 
in table 4.

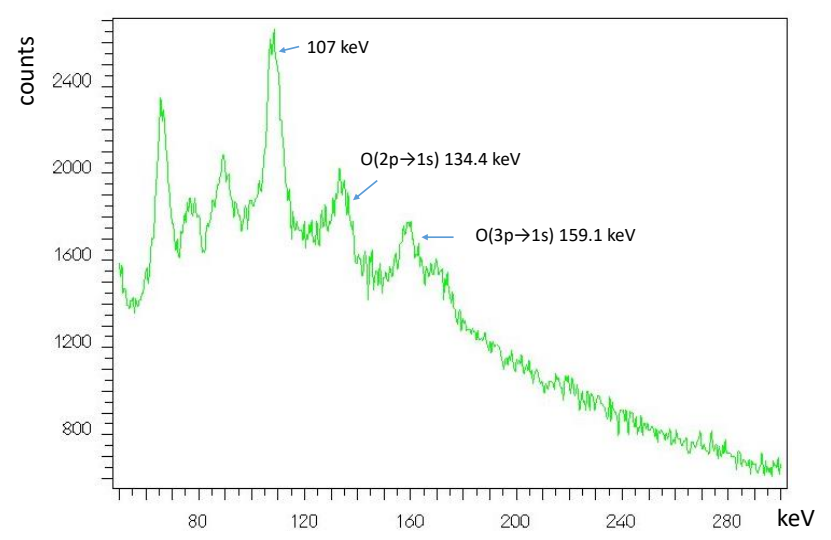

Figure 21. 50-300 keV energy spectrum as seen from the GEM-S HPGe detector with a fast shaping of signals from an Ortec 579 amplifier.

Table 4. Characteristic X-lines as reconstructed by the GEM-S HPGe detector.

\begin{tabular}{|c|c|c|c|c|c|c|c|}
\hline & & \multicolumn{2}{|c|}{ pre amplifier } & \multicolumn{2}{|c|}{ Ortec 672} & \multicolumn{2}{|c|}{ Ortec 579} \\
\hline transition & $\begin{array}{l}\text { Energy }(\mathrm{keV}) \\
\text { (nominal) }\end{array}$ & $\begin{array}{c}\mathrm{E}(\mathrm{keV}) \\
(\mathrm{rec} .)\end{array}$ & FWHM(keV) & $\begin{array}{c}\mathrm{E}(\mathrm{keV}) \\
(\mathrm{rec} .)\end{array}$ & FWHM(keV) & $\begin{array}{c}\mathrm{E}(\mathrm{keV}) \\
(\mathrm{rec} .)\end{array}$ & FWHM(keV) \\
\hline $\mathrm{Al} 3 d \rightarrow 2 p$ & 66.1 & 65.9 & $4.5 \pm 0.1$ & 67.0 & $2.1 \pm 0.1$ & 66.3 & $5.5 \pm 0.1$ \\
\hline C $2 p \rightarrow 1 s$ & 75.3 & 76.8 & $4.5 \pm 0.1$ & 77.2 & $2.1 \pm 0.1$ & 77.2 & $5.5 \pm 0.1$ \\
\hline C $3 p \rightarrow 1 s$ & 89.2 & 88.9 & $4.5 \pm 0.1$ & 89.5 & $2.1 \pm 0.1$ & 89.2 & $5.5 \pm 0.1$ \\
\hline & & 107 & $5.1 \pm 0.4$ & 107 & $2.4 \pm 0.2$ & 107.3 & $5.5 \pm 0.1$ \\
\hline $\mathrm{O} 2 p \rightarrow 1 s$ & 133.5 & 133.9 & $5.4 \pm 0.5$ & 133.4 & $2.7 \pm 0.5$ & 134.4 & $7.4 \pm 0.5$ \\
\hline $\mathrm{O} 3 p \rightarrow 1 s$ & 158.4 & 157.6 & $5.2 \pm 0.4$ & 157.5 & $3.5 \pm 0.5$ & 160 & $4.3 \pm 0.3$ \\
\hline $\mathrm{O} 4 p \rightarrow 1 s$ & 167.1 & - & - & - & - & 170.7 & $8 \pm 1$ \\
\hline $\mathrm{Al} 2 p \rightarrow 1 s$ & 346 & 347 & $6.7 \pm 0.2$ & 345 & $3.1 \pm 0.5$ & 348 & $12 \pm 1$ \\
\hline
\end{tabular}

The timing properties of the HPGe detectors are instead shown in figure 22, for the GLP one. The two peaks structure of the beam is shown in the enlarged inset, where the time parameters for the two beam spills in the beam: FWHM and distance between peaks are well reconstructed.

The time evolution of the $K_{\alpha}(2 p \rightarrow 1 s)$ line in events recorded by the GEM-S HPGe detector for a $\mathrm{H}_{2}+\mathrm{O}_{2}(0.3 \%)$ target, is shown in figure 23. Four successive time slices are shown: 1520 $1720 \mathrm{~ns}$ in panel (a), 1800-2000 ns in panel (b), 2000-2200 ns in panel (c) and last 2200-2400 ns in panel (d).

In conclusion, both types of HPGe detectors have shown in real high-rate and noisy running conditions, a good energy resolution $(\sim 2 \%)$ in the $100 \mathrm{keV}$ region of interest and a good time resolution and are thus able to mitigate problems connected with pile-up events. 


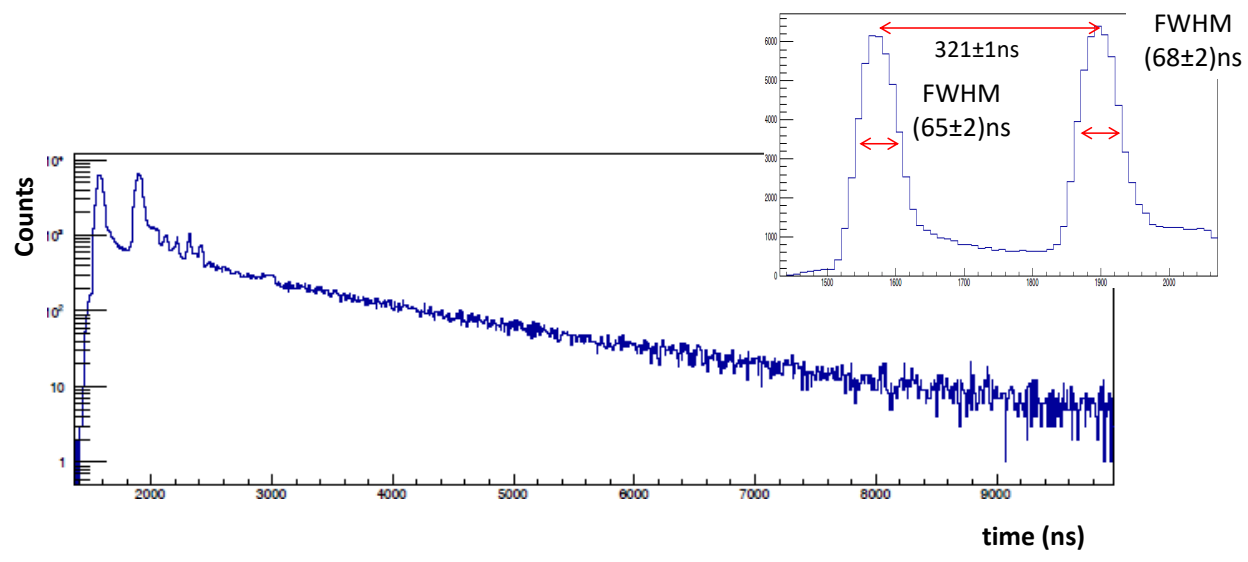

Figure 22. Reconstructed time structure of the beam spills from the GLP HPGe detectors.

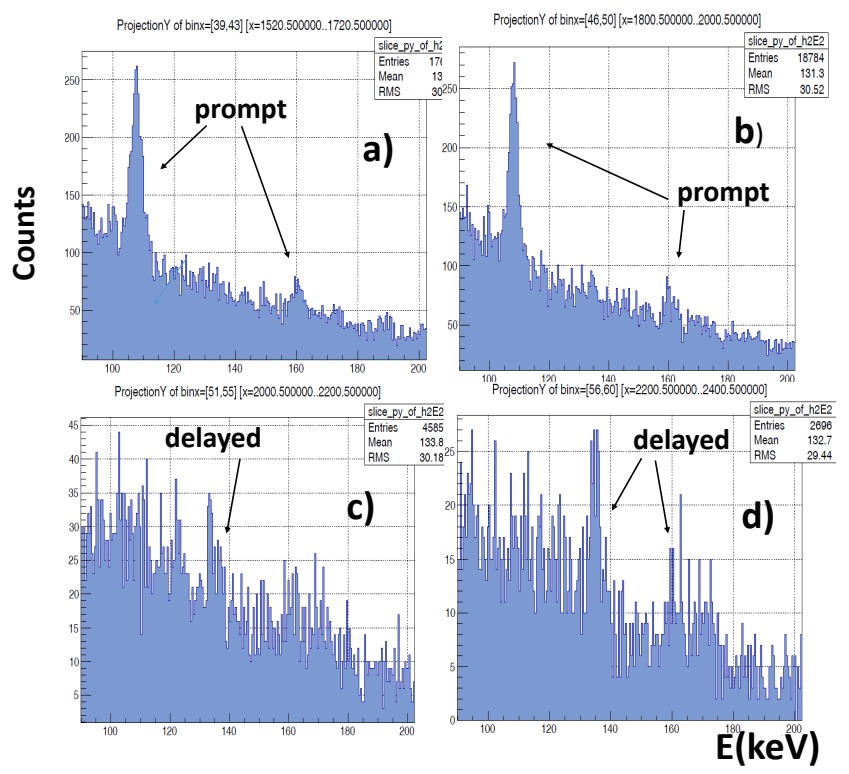

Figure 23. Time evolution of the $K_{\alpha}(2 p \rightarrow 1 s)$ line energy distribution, as reconstructed from the GEMS HPGe detector, in four time slices. (a) 1520-1720 ns, (b) 1800-2000 ns, (c) 2000-2200 ns and (d) 2200-2400 ns.

\section{Conclusions}

The experimental apparatus has been used to study the muon transfer from muonic Hydrogen to the admixed high $\mathrm{Z}$ gases, as Oxygen or Argon. An evolution of this apparatus will be used in the near future to measure the proton Zemach radius of the proton with high precision by measuring the $1 \mathrm{~S}$ muonic hydrogen hyperfine splitting. The setup used in the FAMU experiment at RAL has been described. It includes an high-pressure cryogenic target, a $1 \mathrm{~mm}$ pitch hodoscope for beam 
characterization and a system of fast on purpose developed X-rays detectors, based on Ce: $\mathrm{LaBr}_{3}$ crystals and HPGe detectors. Data have been recorded with a system based on CAEN FADC's in VME standard. Notwithstanding the high counting rate and background conditions, a clean detection of the muonic X-ray lines and a study of their time evolution was possible.

\section{Acknowledgements}

The research activity presented in this paper has been carried out in the framework of the FAMU experiment funded by Istituto Nazionale di Fisica Nucleare (INFN). The use of the low energy muons beam has been allowed by the RIKEN-RAL Muon Facility. We thank the staff of the mechanical workshops of INFN Bologna, Milano Bicocca and Trieste for the continuos and reliable support. We thank the RAL staff (cooling, gas, and radioactive sources sections) and especially Mr. Chris Goodway, Pressure and Furnace Section Leader, for their help, suggestions, professionalism and precious collaboration in the set up of the experiment at RIKEN-RAL.

We thank Criotec srl and especially Ing. Adriano Mussinatto for the technical help and support in the costruction of the FAMU target.

A. Adamczak and D. Bakalov acknowledge the support within the bilateral agreement between the Bulgarian Academy of Sciences and the Polish Academy of Sciences. D. Bakalov, P. Danev and M. Stoilov acknowledge the support of Grant 08-17 of the Bulgarian Science Fund.

We gratefully recognize the help of T. Schneider, CERN EP division, for his support in the optical cutting of the scintillating fibers of the hodoscope detector and the linked problematics, N. Serra from Advansid srl and M. Bombonati from Hamamatsu Photonics Italia srl for useful discussions on SiPM and A. Abba from Nuclear Instruments srl for aid in the problematics of temperature control of SiPM.

\section{References}

[1] A. Vacchi et al., Measuring the size of the proton, SPIE Newsroom (2012); DOI: $10.1117 / 2.1201208 .004274$.

[2] A. Adamczak et al., Hyperfine spectroscopy of muonic hydrogen and the PSI Lamb shift experiment, Nucl. Instr. Meth. B 281 (2012) 7276.

[3] A. Adamczak et al., Atlas of Cross Sections for Scattering of Muonic Hydrogen Atoms on Hydrogen Isotope Molecules, Atomic Data and Nuclear Data Tables 62 (1996) 255.

[4] D. Bakalov et al., Experimental method to measure the hyperfine splitting of muonic hydrogen ( $\left.\mu^{-} p\right)$, Phys. Lett. A 172 (1992) 277.

[5] D. Bakalov et al., Theoretical and computational study of the energy dependence of the muon transfer rate from hydrogen to higher-Z gases, Phys. Lett. A 379 (2015) 151.

[6] T. Matsuzaki et al., The RIKEN-RAL pulsed Muon Facility, Nucl. Instr. Meth. A 465 (2001) 365.

[7] A.Adamczack et al. [FAMU coll.], Steps towards the hyperfine splitting measurement of the muonic hydrogen ground state: pulsed muon beam and detection system characterization, JINST 11/05 (2016) P05007 .

[8] A. Vacchi et al. [FAMU coll.], FAMU experiment: studies of the muon transfer process in a mixture of hydrogen and higher Z gas, RIKEN Accel. Prog. Rep. 49 (2016). 
[9] E. Mocchiutti et al. [FAMU Coll.], First FAMU observation of muon transfer from $\mu p$ atoms to higher-Z elements, JINST 13 (2018) P02019.

[10] R.Carbone et al., the fiber-SIPMT beam monitor of the R484 experiment at RIKEN-RAL muon facility, JINST 10 (2015) C03007.

[11] M. Bonesini et al., The construction of the fiber-SiPM beam monitor system of the R484 and R582 experiment at the RIKEN-RAL muon facility, JINST 12 (2017) C03035.

[12] A. Werthmüller et al., Energy dependence of the charge exchange reaction from muonic hydrogen to oxygen, Hyp. Interact. 116 (1998) 1.

[13] http://www.criotec.com

[14] R. Pani et al., Energy resolution measurements of Ce: $\mathrm{LaBr}_{3}$ scintillating crystals with an ultra-high quantum efficiency photomultiplier tube, Nucl. Instr. Meth. A 610 (2009) 41.

[15] http://www.crystals.saintgobain.com/sites/imdf.crystals.com/files/documents/efficiency_calculations_brochure_69670.pdf

[16] Saint-Gobain Crystals, Brilliance 380 Scintillation Material data sheet and BrilLanCe ${ }^{T M}$ Scintillators Performance Summary, www.detectors.saint-gobain.com.

[17] G. Baldazzi et al., The LaBr 3 based detection system for the FAMU experiment, JINST 12 (2017) C03067.

[18] M. Bonesini et al., Systematic study of innovative hygroscopic and non-hygroscopic crystals with SPM array readout, PoS EPS-HEP2017 (2017) 777.

[19] https://www.ortec-online.com/products/application-software/maestro-mca

[20] S. Agostinelli et al., Geant4-a simulation toolkit, Nucl. Instr. Meth. A 506 (2003) 250.

[21] P. Strasser et al., RIKEN Accel. Prog. Rep. 36 (2003) 202.

[22] M.Soldani et al., High performance DAQ for muon spectroscopy experiments, submitted to Nucl. Instr. Meth. A.

[23] https://root.cern.ch/

[24] M. Tanabashi et al., (Particle Data Group), Phys. Rev. D 98(2018) 030001.

[25] A. Menegolli et al., Performance of X-rays crystal detectors with SiPM array readout exposed to the RIKEN-RAL low energy muon beam, submitted to Nucl. Instr. Meth. A 